\title{
过氧化物诱导自由基串联环化合成苯并咪唑并异喹啉酮反应
}

\author{
王 薪 ${ }^{a}$ 李国锋 ${ }^{c}$ 孙凯*,b 张 冰*,a,b \\ $\left({ }^{a}\right.$ 郑州大学化工学院 郑州 455001) \\ ( ${ }^{b}$ 安阳师范学院化学化工学院 河南安阳 455000) \\ ( ${ }^{c}$ 河南师范大学化学化工学院 河南新乡 453007)
}

\begin{abstract}
摘要 发展了一种过氧化物诱导的 2-芳基苯并咪唑衍生物, 在温和条件下经历自由基环化反应, 一步合成了系列骨架 多样性的苯并咪唑并异咺啉酮化合物的新方法. 该反应具有底物范围宽泛、官能团兼容性好、步骤经济等特点. 机理 研究表明该反应经历了碳中心自由基历程.

关键词 自由基; 碳环化; 多环化合物; 咪唑并异喹啉酮
\end{abstract}

\section{Peroxide-Induced Radical Relay Carbocyclization towards Polycyclic Benzimidazole[2,1-a]isoquinolines}

\author{
Wang, Xin ${ }^{a} \quad$ Li, Guofeng ${ }^{c} \quad$ Sun, Kai ${ }^{*, b} \quad$ Zhang, Bing ${ }^{*, a, b}$ \\ ( ${ }^{a}$ School of Chemical Engineering, Zhengzhou University, Zhengzhou 450001) \\ $\left({ }^{b}\right.$ College of Chemistry and Chemical Engineering, Anyang Normal University, Anyang 455000) \\ ( ${ }^{c}$ School of Chemistry and Chemical Engineering, Henan Normal University, Xinxiang 453007)
}

\begin{abstract}
In this paper, a new peroxide-induced carbon-centered radical relay carbocyclization reaction with 2-arylbenzoimidazoles is described. This method provides an efficient route to a series of structurally diverse benzimidazole $[2,1-a]$ isoquinolines under mild conditions in a straightforward manner. The reaction is compatible with a wide substrate scope, excellent functional group tolerance and high step economy. Mechanistic studies suggest that the reaction proceeds through a carbon-centered radical pathway.

Keywords radical; carbocyclization; polycyclic compounds; benzimidazole[2,1-a]isoquinolines
\end{abstract}

\section{Introduction}

Carbon-centered radicals play important roles in chemical transformations and serve as versatile synthetic intermediates. ${ }^{[1]}$ Therefore, methods for chemical bond formation based on carbon-centered radicals have gained much attention from the scientific community. Carbon-centered radicals relay functionalization, usually proceeding through two or more consecutive steps, and are synthetically attractive as they provide easy access to highly functionalized, often polycyclic, skeletons in a step-economic manner. ${ }^{[2]}$ Structurally diverse polycyclic benzimidazole[2,1- $a$ ] isoquinolines are important synthetic intermediates and key structural motifs in biologically active molecules. ${ }^{[3]}$ Numerous methods have thus been reported toward these carbocyclic frameworks, however, many of these are heavily dependent on multi-step condensation reactions with pre-functionalized starting materials, with limited functional group tolerability and low overall yields. ${ }^{[4]}$ Therefore, investigating more general methods, especially one-pot strategies, to access these frameworks with readily available starting materials is highly desirable. Radical-based carbocyclization reactions, initiated by intermolecular addition of carbon-centered radicals to $\pi$ systems, is undoubtedly good method for the rapid and convenient construction of these frameworks with high atom and step economy. ${ }^{[5]}$

In 2018, an ingenious $\mathrm{Cp} * \mathrm{Rh}(\mathrm{III})$-catalyzed [4+2] annulation for the synthesis of benzimidazole[2,1-a]isoquinolines with 2-arylimidazoles and $a$-diazoketoesters was realized by Song et al. (Scheme 1a). ${ }^{[6]}$ Later, Chen and $\mathrm{Yu}$ et $a l^{[7]}$ reported a silver-catalyzed decarboxylative

* Corresponding authors. E-mail: sunk468@nenu.edu.cn; zhangb@zzu.edu.cn

Received February 27, 2020; revised February 29, 2020; published online March 6, 2020.

Project supported by the National Natural Science Foundation of China (No. 21801007) and the Program for Innovative Research Team of Science and Technology in the University of Henan Province (Nos. 18IRTSTHN004, 18HASTIT006).

国家自然科学基金(No. 21801007)和河南省高校科技创新团队(Nos. 18IRTSTHN004, 18HASTIT006)资助项目. 
carbon-centered radical addition/cyclization and visiblelight-induced radical cyclization to access benzimidazole$[2,1-a]$ isoquinolines (Scheme 1b). However, stoichiometric $\mathrm{acid} /$ base additives, environmentally unfriendly halohydrocarbons and inconvenient inert gas operating requirements, severely limit the synthetic application of these protocols. The "magic methyl effect" has had a profound impact on biological activities, pharmacokinetic profiles, and physical properties, thus, investigation of methods for the direct introduction of methyl groups has attracted much attention from chemists. ${ }^{[8]}$ Along with the widely used alkyl-metallic reagents, alkyl boronates and redox-active esters, the use of peroxides as alternative methylation reagents has also achieved success in many studies. The reaction of di-tert-butyl peroxide (DTBP) with benzaldehyde for the synthesis of diphenylethylene glycol dibenzoate was reported in $1948 .^{[9]}$ Later, the use of dicumyl peroxide (DCP) ${ }^{[10]}$ tert-butyl peroxybenzoate $(\mathrm{TBPB}),{ }^{[1]} \mathrm{DTBP}^{[12]}$ and tert-butyl hydroperoxide (TBHP) ${ }^{[13]}$ as methylation reagents was widely reported. With our continued interest in the derivatization of nitrogen-containing heterocycles ${ }^{[14]}$ and radical chemistry, ${ }^{[15]}$ we thus wondered whether alkyl peroxides could initiate the intermolecular addition of carbon-centered radicals to $\pi$ systems, to achieve a radical relay carbocyclization, which would offer an unprecedented approach to polycyclic benzimidazole[2,1- $a$ ] isoquinolines in a straightforward manner (Scheme 1c).

Previous work:

(a)<smiles>[R]c1ccc(-c2nc3ccccc3[nH]2)cc1</smiles>

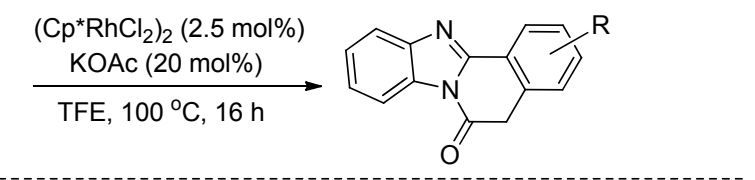

(b)<smiles>C=C(C)C(=O)n1c(-c2ccc(I)cc2)nc2ccccc21</smiles>
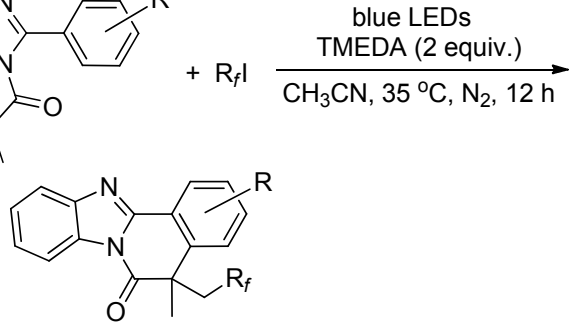

This study:

(c)<smiles>[R]c1ccc(-c2nc3ccccc3n2C(=O)C(=C)C)cc1</smiles><smiles>[R]OOC(=O)c1ccccc1</smiles>

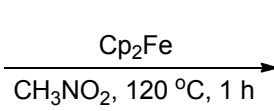<smiles>[R]CC1(C[R])C(=O)n2c(nc3ccccc32)-c2ccc([R])cc21</smiles>

Scheme 1 Synthesis of benzimidazo-isoquinoline frameworks

\section{Results and discussion}

We initiated our studies by screening the reaction conditions with $N$-methacryloyl-2-phenylbenzoimidazole (1a) as substrate and various peroxides as methylation reagents (Table 1). The experiments revealed that DCP showed low reactivity, while TBHP and DTBP failed to afford the desired product 2a (Entries 1 3). It was found that TBPB was a good choice, and $\mathbf{2 a}$ was obtained in $51 \%$ yield (Entry 4). Therefore, the reaction was then carried out in different solvents, including DMSO, $\mathrm{CH}_{3} \mathrm{NO}_{2}, \mathrm{THF}, \mathrm{DCE}$ and EtOAc, to optimize the yield. The most favorable solvent was found to be $\mathrm{CH}_{3} \mathrm{NO}_{2}$, leading to an evident improvement in the yield of $\mathbf{2 a}(63 \%)$ (Entries 5 9). In addition, parallel reactions with different additives such as $\mathrm{FeCl}_{2}, \mathrm{FeCl}_{3}, \mathrm{Fe}(\mathrm{OTf})_{3}$ and ferrocene $\left(\mathrm{Cp}_{2} \mathrm{Fe}\right)$ were carried out, and $\mathrm{Cp}_{2} \mathrm{Fe}$ was found to be the best promoter, affording $2 \mathbf{a}$ in up to $84 \%$ yield (Entries 10 13). Decreasing the loading of $\mathrm{Cp}_{2} \mathrm{Fe}$ from $20 \%$ to $10 \%$ had no obvious effect on yield of $\mathbf{2 a}$, however, the yield was slightly lower with $5 \% \mathrm{Cp}_{2} \mathrm{Fe}$ added (Entries 14 16). Further examination on the impact of reaction time and temperature showed that 1 $\mathrm{h}$ was enough to achieve the formation of $\mathbf{2 a}$ with a high yield, while a decrease in the yield was observed when the reaction temperature was decreased from $120{ }^{\circ} \mathrm{C}$ to $90{ }^{\circ} \mathrm{C}$ and $60{ }^{\circ} \mathrm{C}$ (Entries 17 19).

Table 1 Survey of the reaction conditions ${ }^{a, b}$<smiles>C=C(C)C(=O)n1c(-c2ccccc2)nc2ccccc21</smiles>

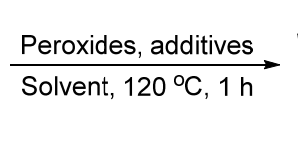

$1 \mathrm{a}$

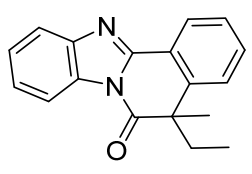

2a

\begin{tabular}{|c|c|c|c|c|}
\hline Entry & Oxidant & Catalyst (mol\%) & Solvent & Yield $/ \%$ \\
\hline 1 & DCP & None & $\mathrm{CH}_{3} \mathrm{CN}$ & 23 \\
\hline 2 & TBHP & None & $\mathrm{CH}_{3} \mathrm{CN}$ & Trace \\
\hline 3 & DTBP & None & $\mathrm{CH}_{3} \mathrm{CN}$ & 0 \\
\hline 4 & ТВРB & None & $\mathrm{CH}_{3} \mathrm{CN}$ & 51 \\
\hline 5 & ТВРВ & None & DMSO & 23 \\
\hline 6 & ТВРВ & None & $\mathrm{CH}_{3} \mathrm{NO}_{2}$ & 63 \\
\hline 7 & ТВРВ & None & THF & 0 \\
\hline 8 & ТВРB & None & DCE & 39 \\
\hline 9 & ТВРВ & None & EtOAc & 0 \\
\hline 10 & ТВРB & $\mathrm{FeCl}_{2}(20)$ & $\mathrm{CH}_{3} \mathrm{NO}_{2}$ & 45 \\
\hline 11 & ТВРВ & $\mathrm{FeCl}_{3}(20)$ & $\mathrm{CH}_{3} \mathrm{NO}_{2}$ & 26 \\
\hline 12 & ТВРВ & $\mathrm{Fe}(\mathrm{OTf})_{3}(20)$ & $\mathrm{CH}_{3} \mathrm{NO}_{2}$ & Trace \\
\hline 13 & ТВРВ & $\mathrm{Cp}_{2} \mathrm{Fe}(20)$ & $\mathrm{CH}_{3} \mathrm{NO}_{2}$ & 84 \\
\hline 14 & ТВРВ & $\mathrm{Cp}_{2} \mathrm{Fe}(15)$ & $\mathrm{CH}_{3} \mathrm{NO}_{2}$ & 82 \\
\hline 15 & ТВРB & $\mathrm{Cp}_{2} \mathrm{Fe}(10)$ & $\mathrm{CH}_{3} \mathrm{NO}_{2}$ & 83 \\
\hline 16 & TBPB & $\mathrm{Cp}_{2} \mathrm{Fe}(5)$ & $\mathrm{CH}_{3} \mathrm{NO}_{2}$ & 74 \\
\hline 17 & ТВРВ & $\mathrm{Cp}_{2} \mathrm{Fe}(10)$ & $\mathrm{CH}_{3} \mathrm{NO}_{2}$ & $80^{c}$ \\
\hline 18 & TBPB & $\mathrm{Cp}_{2} \mathrm{Fe}(10)$ & $\mathrm{CH}_{3} \mathrm{NO}_{2}$ & $45^{d}$ \\
\hline 19 & TBPB & $\mathrm{Cp}_{2} \mathrm{Fe}(10)$ & $\mathrm{CH}_{3} \mathrm{NO}_{2}$ & $11^{e}$ \\
\hline
\end{tabular}

${ }^{a}$ Reactions were carried out with $\mathbf{1 a}(0.3 \mathrm{mmol})$, peroxides $(2.0$ equiv. $)$, catalyst $(5 \sim 20 \mathrm{~mol} \%)$ and solvent $(2 \mathrm{~mL})$ refluxed at $120{ }^{\circ} \mathrm{C}$ for $1 \mathrm{~h} .{ }^{b}$ Yield of the isolated product. ${ }^{c}$ Reaction performed at $120{ }^{\circ} \mathrm{C}$ for $6 \mathrm{~h} .{ }^{d}$ Reaction performed at $90{ }^{\circ} \mathrm{C}$ for $6 \mathrm{~h} .{ }^{e}$ Reaction performed at $60{ }^{\circ} \mathrm{C}$ for $6 \mathrm{~h}$. 
With the optimal reaction conditions, the substrate scope was explored as shown in Table 2. A wide range of functional groups, including electron-withdrawing and electron-donating groups at the ortho-, meta- and pa$r a$-positions of the phenyl rings, were compatible with the reaction conditions, giving the corresponding products $\mathbf{2 b} \sim \mathbf{2 n}$ in moderate to excellent yields $(53 \% \sim 91 \%)$. In this reaction, halo-substituents $(\mathrm{Cl}, \mathrm{Br}$ and $\mathrm{I})$ on the phenyl ring, provide a handle for further transformations. Strongly electron-withdrawing groups $\left(\mathrm{CF}_{3}, \mathrm{COOMe}, \mathrm{SO}_{2} \mathrm{Me}\right)$, which are negative to the radical carbocyclization, underwent smoothly the reaction to give $\mathbf{2 l} \sim \mathbf{2 n}$ in good yields. Notably, in place of a benzene ring, 2-thiophen-substituted substrate 1o, which is usually sensitive in oxidizing radical coupling systems, was well tolerated, and the corresponding product 20 was obtained in reasonable yield (63\%). In addition, 2-naphthalen-substituted $1 H$-benzo $[d]$ imidazole $\mathbf{1 p}$ and polysubstituted substrates $\mathbf{1 q}$ and $\mathbf{1 r}$, were also smoothly converted to the corresponding products $\mathbf{2 p}, \mathbf{2 q}$ and $2 \mathrm{r}$ in moderate to good yields $(55 \% \sim 82 \%)$.

With the developed strategy in hand, benzoyl peroxide (BPO), another phenyl radical precursor, ${ }^{[16]}$ was reacted with substrates $\mathbf{1}$, with the hope of constructing some other structurally diverse benzimidazole $[2,1-a]$ isoquinolines. After screening of reaction conditions, contrary to the pre- vious system, it was found that $\mathrm{Cp}_{2} \mathrm{Fe}$ did not require in this reaction. The scope of substrates $\mathbf{1}$ was briefly tested and the results are listed in Table 3. Substrates bearing electron-neutral $(\mathrm{H})$ and electron-donating (Me and $\mathrm{OMe}$ ) groups on the phenyl rings proceeded smoothly under the optimal conditions. The desired products $\mathbf{3 a} \sim \mathbf{3 e}$ were obtained in moderate to good yields $(60 \% \sim 79 \%)$. Obvious electronic effects were observed with substrates bearing electron-withdrawing groups $(\mathrm{F}, \mathrm{Cl}, \mathrm{Br}$ and $\mathrm{CN})$ on the phenyl rings, affording the corresponding products $\mathbf{3 f} \sim \mathbf{3 j}$ in relatively low yields $(30 \% \sim 53 \%)$.

Several control experiments were conducted to gain insights into the reaction mechanism. When the model reaction was treated with radical scavenger 2,2,6,6-tetramethylpiperidine-1-oxyl (TEMPO, 2 equiv), the yield of $\mathbf{2 a}$ decreased to $13 \%$ (Eq. 1). When 2,6-di-tert-butyl-4-methylphenol (BHT, 2 equiv.) and 1,1-diphenylethylene (2 equiv) were added under standard conditions, the reactions were totally suppressed, and the adducts $\mathbf{4}$ and $\mathbf{5}$ were detected via GC-MS analysis (Eqs. 3, 4). The above experiments supported a radical relay carbocyclization pathway. With the present findings and previous reports, ${ }^{[17]}$ a possible radical relay carbocyclization mechanism is proposed in Scheme 2.

Table 2 Substrate scope $\mathrm{e}^{a, b}$
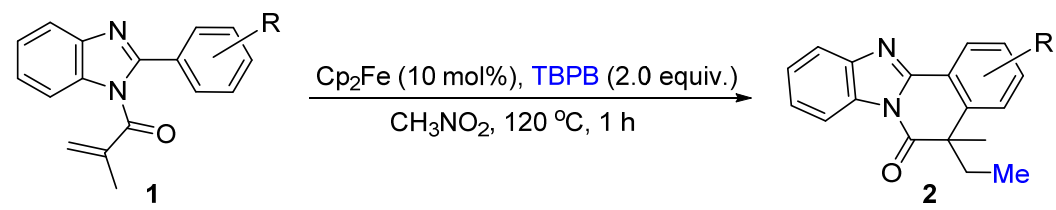

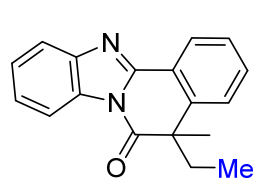

2a, $83 \%$

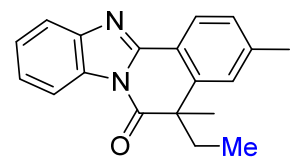

2g, $91 \%$

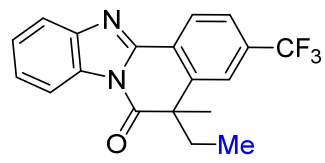

2I, $76 \%$

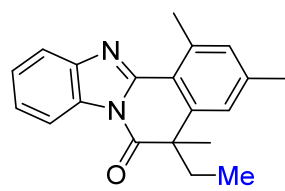

2q, $67 \%$

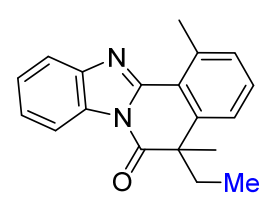

2b, $69 \%$

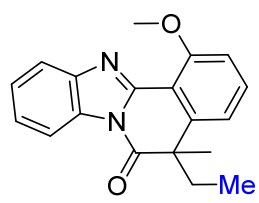

2c, $83 \%$

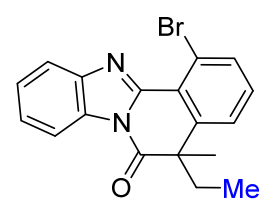

2d, $53 \%$

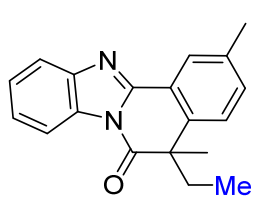

2e, $72 \%$

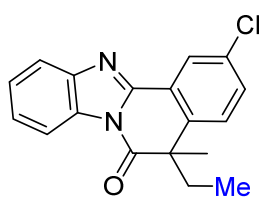

2f, $71 \%$

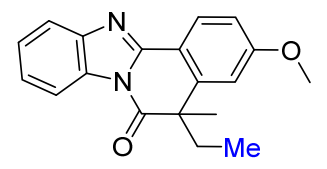

2h, $79 \%$

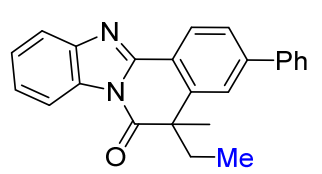

2i, $60 \%$

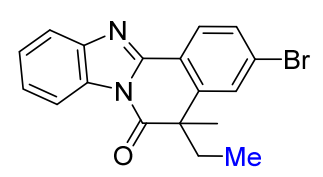

2j, $64 \%$

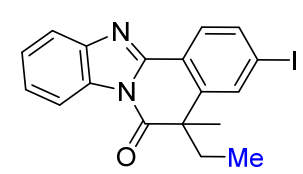

2k, $52 \%$

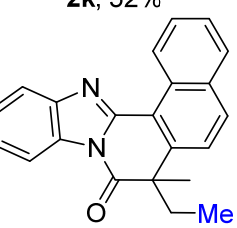

2p, $82 \%$

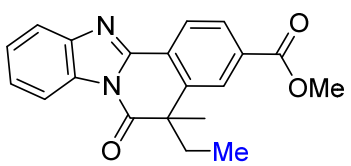

2m, 83\%

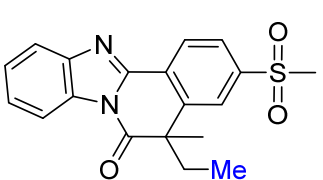

2n, $81 \%$

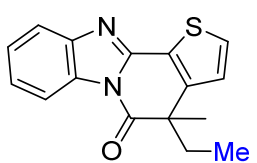

2o, $63 \%$

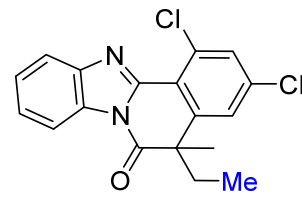

2r, 55\%

${ }^{a}$ Reactions were carried out with 1 ( $\left.0.3 \mathrm{mmol}\right)$, TBPB (2.0 equiv.), and $\mathrm{Cp}_{2} \mathrm{Fe}(10 \mathrm{~mol} \%)$ in $\mathrm{CH}_{3} \mathrm{NO}_{2}(2 \mathrm{~mL})$ refluxed at $120{ }^{\circ} \mathrm{C}$ for $1 \mathrm{~h} .{ }^{b}$ Yield of isolated product. 
Table 3 Substrate scope $\mathrm{e}^{a, b}$

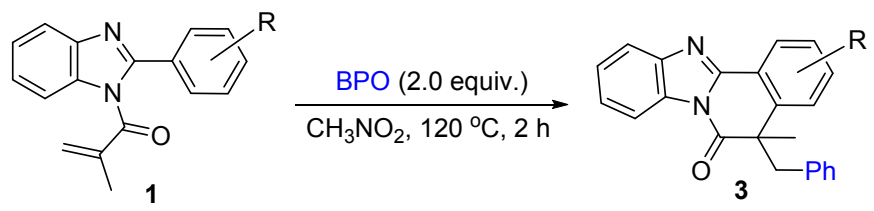<smiles>CC1(Cc2ccccc2)C(=O)n2c(nc3ccccc32)-c2ccccc21</smiles>

3a, $60 \%$

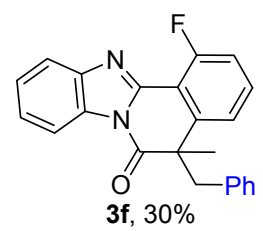

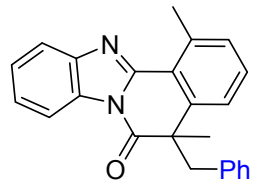

3b, $61 \%$

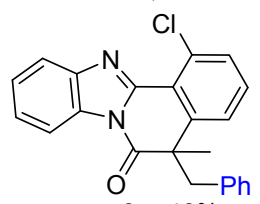

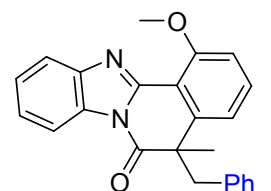

3c, $76 \%$

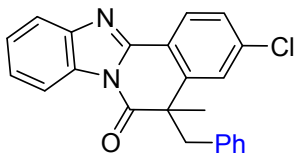

$3 \mathrm{~h}, 53 \%$

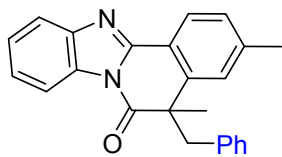

3d, $69 \%$

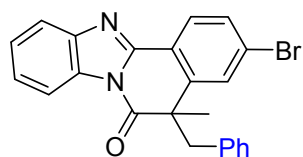

3i, $45 \%$

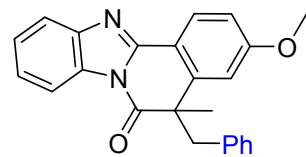

3 e, $79 \%$

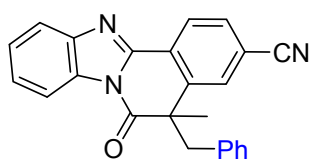

3j, $40 \%$

${ }^{a}$ Reactions were carried out with $1(0.3 \mathrm{mmol})$ and $\mathrm{BPO}\left(2.0\right.$ equiv) in $\mathrm{CH}_{3} \mathrm{NO}_{2}(2 \mathrm{~mL})$ refluxed at $120{ }^{\circ} \mathrm{C}$ for $2 \mathrm{~h} .{ }^{b}$ Yield of isolated product.<smiles>C=C(C)C(=O)n1c(-c2ccccc2)nc2ccccc21</smiles>

$1 \mathrm{a}$

$\mathrm{Cp}_{2} \mathrm{Fe}(10 \mathrm{~mol} \%)$

TBPB (2.0 equiv.)

TEMPO (2.0 equiv.)

$\mathrm{CH}_{3} \mathrm{NO}_{2}, 120^{\circ} \mathrm{C}, 1 \mathrm{~h}$

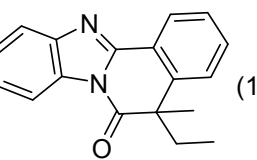<smiles>C=C(C)C(=O)n1c(-c2ccccc2)nc2ccccc21</smiles>

$\mathrm{Cp}_{2} \mathrm{Fe}(10 \mathrm{~mol} \%)$ TBPB (2.0 equiv.) $\underset{\mathrm{CH}_{3} \mathrm{NO}_{2}, 120^{\circ} \mathrm{C}, 1 \mathrm{~h}}{\mathrm{BHT}(2.0 \text { equiv. }}$<smiles>COc1c(C(C)(C)C)cc(C)cc1C(C)(C)C</smiles>

4, detected by GC-MS detection<smiles>C=C(C)C(=O)n1c(-c2ccccc2)nc2ccccc21</smiles>

1a<smiles>CC=C(c1ccccc1)c1ccccc1</smiles>

2a, trace $\mathrm{p}_{2} \mathrm{Fe}(10 \mathrm{~mol} \%)$

$\overrightarrow{\text { 1,1-diphenylethylene }}$ (2.0 equiv.) $\mathrm{CH}_{3} \mathrm{NO}_{2}, 120^{\circ} \mathrm{C}, 1 \mathrm{~h}$
5, detected by GC-MS detection

The reaction begins with the $\mathrm{O}-\mathrm{O}$ bond cleavage of peroxides, providing $O$-centered radical species A. Further splitting of $\mathbf{A}$ by loss of one equivalent of acetone or carbon dioxide gives the free methyl or phenyl radical species B. In the presence of $\mathrm{Fe}(\mathrm{II})$, the generation of the tertbutoxy radical may be accelerated by a single electron transfer (SET) process, along with release the Fe(III) species. Then, the addition of radical species B to $N$-methacryloyl-2-phenyl-benzoimidazoles (1) provides another radical intermediate $\mathbf{C}$, which undergoes an intramolecular relay cyclization to give the radical intermediate $\mathbf{D}$. Subsequent reaction of intermediate $\mathbf{D}$ with the other $O$-centered radical species $\mathbf{A}$ or the Fe(III) species generates a cationic intermediate $\mathbf{E}$, then loss of a proton from $\mathbf{E}$ eventually affords the desired products $\mathbf{2}$ and $\mathbf{3}$.

\section{Conclusions}

In summary, we have developed a simple and efficient

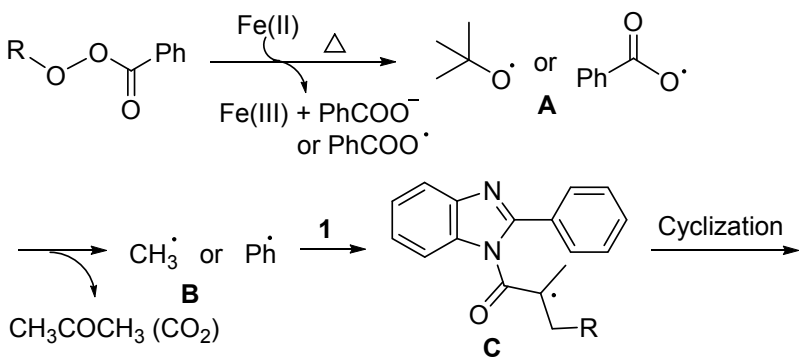

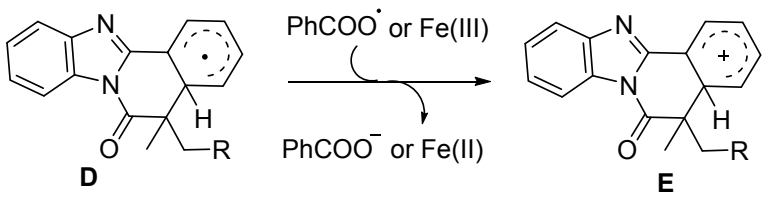<smiles>[R]CC1(C)C(=O)n2c(nc3ccccc32)-c2ccccc21</smiles>

Scheme 2 Plausible catalytic cycle

peroxide-induced radical relay carbocyclization reaction from easily available $N$-methacryloyl-2-phenylbenzoimidazoles to assemble a series of structurally diverse and privileged polycyclic benzimidazo[2,1-a]isoquinoline-6$(5 H)$-ones in a straightforward manner. Furthermore, this reaction features simple reaction conditions, broad substrate scope, good functional group tolerance and easyhandling. This mild radical relay carbocyclization strategy has inspired an interest in the synthesis of other polycyclic compounds, and related investigations are currently underway in our laboratory.

\section{Experimental section}

\subsection{Instruments and reagents}

All reagents were purchased from commercial sources 
and used without further purification. ${ }^{1} \mathrm{H}$ NMR and ${ }^{13} \mathrm{C}$ NMR spectra were recorded on a Bruker Ascend ${ }^{\mathrm{TM}} 400$ spectrometer in deuterated solvents containing TMS as an internal reference standard. High-resolution mass spectrometry (HRMS) analyses were conducted on a Waters LCT Premier/XE. Melting points were measured on a melting point apparatus equipped with a thermometer and were uncorrected. All the reactions were monitored by thin-layer chromatography (TLC) using GF254 silica gelcoated TLC plates. Purification by flash column chromatography was performed over $\mathrm{SiO}_{2}$ (silica gel 200 300 mesh).

\subsection{General procedure for the synthesis of 2}

2-Methyl-1-(2-phenyl-1H-benzo[d]imidazol-1-yl)prop-2en-1-one (1a) (78.7 mg, $0.3 \mathrm{mmol})$, ferrocene (5.6 mg, 10 mol\%) and TBPB (114 $\mu \mathrm{L}, 2.0$ equiv.) were mixed in 2 $\mathrm{mL}$ of $\mathrm{CH}_{3} \mathrm{NO}_{2}$, and then the mixture was refluxed at $120{ }^{\circ} \mathrm{C}$ in oil bath for $1 \mathrm{~h}$. After completion of the reaction, the mixture was quenched by $\mathrm{NaHCO}_{3}$ (sat. aq. 15 $\mathrm{mL})$ and extracted with $\mathrm{CH}_{2} \mathrm{Cl}_{2}(5 \mathrm{~mL} \times 3)$. Then the organic solvent was concentrated in vacuo. The residue was purified by flash column chromatography with petroleum ether/ethyl acetate $(V: V=10: 1)$ as eluent to give 5ethyl-5-methylbenzo[4,5]imidazo[2,1- $a$ ] isoquinolin-6(5H)one (2a) (83\% yield, $68.8 \mathrm{mg})$. White solid, m.p. 57 $58{ }^{\circ} \mathrm{C} ;{ }^{1} \mathrm{H}$ NMR $\left(400 \mathrm{MHz}, \mathrm{CDCl}_{3}\right) \delta: 8.48(\mathrm{~d}, J=8.0 \mathrm{~Hz}$, $1 \mathrm{H}), 8.41 \sim 8.33(\mathrm{~m}, 1 \mathrm{H}), 7.87 \sim 7.77(\mathrm{~m}, 1 \mathrm{H}), 7.62 \sim 7.54$ $(\mathrm{m}, 1 \mathrm{H}), 7.53 \sim 7.38(\mathrm{~m}, 4 \mathrm{H}), 2.51 \sim 2.38(\mathrm{~m}, 1 \mathrm{H}), 2.09 \sim$ $1.96(\mathrm{~m}, 1 \mathrm{H}), 1.73(\mathrm{~s}, 3 \mathrm{H}), 0.58(\mathrm{t}, J=7.4 \mathrm{~Hz}, 3 \mathrm{H}) ;{ }^{13} \mathrm{C}$ NMR $\left(100 \mathrm{MHz}, \mathrm{CDCl}_{3}\right) \delta: 173.49,150.09,144.14$, $141.69,132.00,131.39,127.79,126.19,125.98,125.93$, $125.65,123.33,119.87,115.80,50.18,36.51,28.48,9.70$. HRMS (ESI) calcd for $\mathrm{C}_{18} \mathrm{H}_{17} \mathrm{~N}_{2} \mathrm{O}[\mathrm{M}+\mathrm{H}]^{+} 277.1335$, found 277.1339 .

5-Ethyl-1,5-dimethylbenzo[4,5]imidazo[2,1- $a$ ]isoquinolin-6(5H)-one (2b): White solid. Isolated by flash column chromatography (petroleum ether/ethyl acetate, $V: V=$ $20: 1)$ as eluent to give $2 \mathbf{b}(69 \%$ yield, $60 \mathrm{mg})$. m.p. $94 \sim$ $95{ }^{\circ} \mathrm{C} ;{ }^{1} \mathrm{H}$ NMR $\left(400 \mathrm{MHz}, \mathrm{CDCl}_{3}\right) \delta: 8.44 \sim 8.37(\mathrm{~m}$, $1 \mathrm{H}), 7.88 \sim 7.81(\mathrm{~m}, 1 \mathrm{H}), 7.48 \sim 7.38(\mathrm{~m}, 3 \mathrm{H}), 7.38 \sim 7.28$ $(\mathrm{m}, 2 \mathrm{H}), 3.06(\mathrm{~s}, 3 \mathrm{H}), 2.50 \sim 2.36(\mathrm{~m}, 1 \mathrm{H}), 2.07 \sim 1.94(\mathrm{~m}$, 1H), $1.73(\mathrm{~s}, 3 \mathrm{H}), 0.58(\mathrm{t}, J=7.4 \mathrm{~Hz}, 3 \mathrm{H}) ;{ }^{13} \mathrm{C}$ NMR $(100$ $\left.\mathrm{MHz}, \mathrm{CDCl}_{3}\right) \delta: 173.64,150.28,144.32,142.79,139.76$, $131.05,130.63,130.57,125.67,124.04,122.03,120.17$, $115.80,50.06,36.92,28.73,24.80,9.69$. HRMS (ESI) calcd for $\mathrm{C}_{19} \mathrm{H}_{19} \mathrm{~N}_{2} \mathrm{O}[\mathrm{M}+\mathrm{H}]^{+}$291.1492, found 291.1489.

5-Ethyl-1-methoxy-5-methylbenzo[4,5]imidazo[2,1-a]isoquinolin-6(5H)-one (2c): Yellow solid. Isolated by flash column chromatography (petroleum ether/ethyl acetate, $V: V=5: 1)$ as eluent to give $2 \mathrm{c}(83 \%$ yield, $76 \mathrm{mg})$. m.p. $155 \sim 156{ }^{\circ} \mathrm{C} ;{ }^{1} \mathrm{H}$ NMR $\left(400 \mathrm{MHz}, \mathrm{CDCl}_{3}\right) \delta: 8.44 \sim$ $8.30(\mathrm{~m}, 1 \mathrm{H}), 7.95 \sim 7.81(\mathrm{~m}, 1 \mathrm{H}), 7.48(\mathrm{t}, J=8.1 \mathrm{~Hz}, 1 \mathrm{H})$, $7.43 \sim 7.33(\mathrm{~m}, 2 \mathrm{H}), 7.07(\mathrm{~d}, J=7.9 \mathrm{~Hz}, 1 \mathrm{H}), 7.01(\mathrm{~d}, J=$ $8.3 \mathrm{~Hz}, 1 \mathrm{H}), 4.10(\mathrm{~s}, 3 \mathrm{H}), 2.45 \sim 2.32(\mathrm{~m}, 1 \mathrm{H}), 2.03 \sim 1.89$ $(\mathrm{m}, 1 \mathrm{H}), 1.71(\mathrm{~s}, 3 \mathrm{H}), 0.55(\mathrm{t}, J=7.3 \mathrm{~Hz}, 3 \mathrm{H}) ;{ }^{13} \mathrm{C} \mathrm{NMR}$ $\left(100 \mathrm{MHz}, \mathrm{CDCl}_{3}\right) \delta: 173.28,158.62,148.07,144.38$,
$144.26,132.30,130.18,125.63,125.54,120.48,118.39$, $115.45,112.59,110.24,56.63,49.89,36.96,28.45,9.53$. HRMS (ESI) calcd for $\mathrm{C}_{19} \mathrm{H}_{19} \mathrm{~N}_{2} \mathrm{O}_{2}[\mathrm{M}+\mathrm{H}]^{+} 307.1441$, found 307.1449 .

1-Bromo-5-ethyl-5-methylbenzo[4,5]imidazo[2,1-a]isoquinolin-6(5H)-one (2d): White solid. Isolated by flash column chromatography (petroleum ether/ethyl acetate, $V: V=20: 1)$ as eluent to give $2 \mathbf{d}(53 \%$ yield, $56 \mathrm{mg})$. m.p. $182 \sim 183{ }^{\circ} \mathrm{C} ;{ }^{1} \mathrm{H}$ NMR $\left(400 \mathrm{MHz}, \mathrm{CDCl}_{3}\right) \delta: 8.45 \sim$ $8.32(\mathrm{~m}, 1 \mathrm{H}), 7.98 \sim 7.88(\mathrm{~m}, 1 \mathrm{H}), 7.81(\mathrm{~d}, J=7.8 \mathrm{~Hz}$, $1 \mathrm{H}), 7.53 \sim 7.40(\mathrm{~m}, 3 \mathrm{H}), 7.35(\mathrm{t}, J=7.9 \mathrm{~Hz}, 1 \mathrm{H}), 2.49 \sim$ $2.34(\mathrm{~m}, 1 \mathrm{H}), 2.05 \sim 1.92(\mathrm{~m}, 1 \mathrm{H}), 1.73(\mathrm{~s}, 3 \mathrm{H}), 0.58(\mathrm{t}$, $J=7.4 \mathrm{~Hz}, 3 \mathrm{H}) ;{ }^{13} \mathrm{C} \mathrm{NMR}\left(100 \mathrm{MHz}, \mathrm{CDCl}_{3}\right) \delta: 172.48$, $147.51,144.75,143.74,135.22,131.33,130.76,126.40$, $125.95,125.70,122.81,121.40,120.87,115.73,50.53$, 36.97, 28.42, 9.63. HRMS (ESI) calcd for $\mathrm{C}_{18} \mathrm{H}_{16} \mathrm{BrN}_{2} \mathrm{O}$ $[\mathrm{M}+\mathrm{H}]^{+}$355.0441, found 355.0444.

5-Ethyl-2,5-dimethylbenzo[4,5]imidazo[2,1-a]isoquinolin-6(5H)-one (2e): White solid. Isolated by by flash column chromatography (petroleum ether/ethyl acetate, $V$ : $V=20: 1)$ as eluent to give $2 \mathrm{e}(72 \%$ yield, $62 \mathrm{mg})$. m.p. $113 \sim 114{ }^{\circ} \mathrm{C} ;{ }^{1} \mathrm{H}$ NMR (400 MHz, $\mathrm{CDCl}_{3}$ ) $\delta: 8.40 \sim 8.34$ $(\mathrm{m}, 1 \mathrm{H}), 8.30(\mathrm{~s}, 1 \mathrm{H}), 7.85 \sim 7.78(\mathrm{~m}, 1 \mathrm{H}), 7.47 \sim 7.33(\mathrm{~m}$, $4 \mathrm{H}), 2.48 \sim 2.38(\mathrm{~m}, 4 \mathrm{H}), 2.05 \sim 1.94(\mathrm{~m}, 1 \mathrm{H}), 1.71(\mathrm{~s}$, $3 \mathrm{H}), 0.57$ (t, $J=7.4 \mathrm{~Hz}, 3 \mathrm{H}) ;{ }^{13} \mathrm{C}$ NMR $\left(100 \mathrm{MHz}, \mathrm{CDCl}_{3}\right)$ $\delta: 173.72,150.30,144.14,138.82,137.71,133.11,131.42$, $126.11,126.02,125.93,125.56,123.04,119.79,115.79$, 49.93, 36.44, 28.55, 21.05, 9.69. HRMS (ESI) calcd for $\mathrm{C}_{19} \mathrm{H}_{19} \mathrm{~N}_{2} \mathrm{O}[\mathrm{M}+\mathrm{H}]^{+}$291.1492, found 291.1497.

2-Chloro-5-ethyl-5-methylbenzo[4,5]imidazo[2,1-a]isoquinolin-6(5H)-one (2f): White solid. Isolated by flash column chromatography (petroleum ether/ethyl acetate, $V: V=20: 1)$ as eluent to give $2 \mathbf{f}(71 \%$ yield, $66 \mathrm{mg})$. m.p. $135 \sim 136{ }^{\circ} \mathrm{C} ;{ }^{1} \mathrm{H}$ NMR $\left(400 \mathrm{MHz}, \mathrm{CDCl}_{3}\right) \delta: 8.47$ $(\mathrm{d}, J=2.0 \mathrm{~Hz}, 1 \mathrm{H}), 8.41 \sim 8.31(\mathrm{~m}, 1 \mathrm{H}), 7.88 \sim 7.76(\mathrm{~m}$, $1 \mathrm{H}), 7.53(\mathrm{dd}, J=8.5,1.9 \mathrm{~Hz}, 1 \mathrm{H}), 7.49 \sim 7.43(\mathrm{~m}, 2 \mathrm{H})$, $7.40(\mathrm{~d}, J=8.5 \mathrm{~Hz}, 1 \mathrm{H}), 2.53 \sim 2.37(\mathrm{~m}, 1 \mathrm{H}), 2.05 \sim 1.92$ $(\mathrm{m}, 1 \mathrm{H}), 1.72(\mathrm{~s}, 3 \mathrm{H}), 0.58(\mathrm{t}, J=7.4 \mathrm{~Hz}, 3 \mathrm{H}) ;{ }^{13} \mathrm{C} \mathrm{NMR}$ $\left(100 \mathrm{MHz}, \mathrm{CDCl}_{3}\right) \delta: 172.89,148.75,144.02,139.93$, $134.04,132.01,131.39,127.83,126.20,126.06,125.57$, $124.97,120.10,115.84,50.07,36.42,28.41,9.68$. HRMS (ESI) calcd for $\mathrm{C}_{18} \mathrm{H}_{16} \mathrm{ClN}_{2} \mathrm{O}[\mathrm{M}+\mathrm{H}]^{+}$311.0946, found 311.0940 .

5-Ethyl-3,5-dimethylbenzo[4,5]imidazo[2,1- $a$ ]isoquinolin-6(5H)-one $(\mathbf{2 g})$ : White solid. Isolated by flash column chromatography (petroleum ether/ethyl acetate, $V: V=$ $20: 1)$ as eluent to give $\mathbf{2 g}$ (91\% yield, $79 \mathrm{mg})$. m.p. $144 \sim 145{ }^{\circ} \mathrm{C} ;{ }^{1} \mathrm{H}$ NMR $\left(400 \mathrm{MHz}, \mathrm{CDCl}_{3}\right) \delta: 8.43 \sim 8.30$ $(\mathrm{m}, 2 \mathrm{H}), 7.80(\mathrm{dd}, J=6.5,2.0 \mathrm{~Hz}, 1 \mathrm{H}), 7.47 \sim 7.37(\mathrm{~m}$, $2 \mathrm{H}), 7.30(\mathrm{~d}, J=8.1 \mathrm{~Hz}, 1 \mathrm{H}), 7.25(\mathrm{~s}, 1 \mathrm{H}), 2.55 \sim 2.36(\mathrm{~m}$, 4H), $2.08 \sim 1.94(\mathrm{~m}, 1 \mathrm{H}), 1.72(\mathrm{~s}, 3 \mathrm{H}), 0.58(J, J=7.4 \mathrm{~Hz}$, $3 \mathrm{H}) ;{ }^{13} \mathrm{C} \mathrm{NMR}\left(100 \mathrm{MHz}, \mathrm{CDCl}_{3}\right) \delta: 173.64,150.34$, $144.22,142.54,141.72,131.36,128.89,126.59,125.89$, $125.87,125.39,120.70,119.69,115.73,50.10,36.55$, 28.50, 22.12, 9.73. HRMS (ESI) calcd for $\mathrm{C}_{19} \mathrm{H}_{19} \mathrm{~N}_{2} \mathrm{O}$ $[\mathrm{M}+\mathrm{H}]^{+}$291.1492, found 291.1490.

5-Ethyl-3-methoxy-5-methylbenzo[4,5]imidazo[2,1- $a$ ]iso- 
quinolin-6(5H)-one (2h): White solid. Isolated by flash column chromatography (petroleum ether/ethyl acetate, $V: V=20: 1)$ as eluent to give $\mathbf{2 h}(79 \%$ yield, $72 \mathrm{mg})$. m.p. 82 83 ${ }^{\circ} \mathrm{C} ;{ }^{1} \mathrm{H}$ NMR $\left(400 \mathrm{MHz}, \mathrm{CDCl}_{3}\right) \delta: 8.41(\mathrm{~d}$, $J=8.7 \mathrm{~Hz}, 1 \mathrm{H}), 8.37 \sim 8.28(\mathrm{~m}, 1 \mathrm{H}), 7.83 \sim 7.72(\mathrm{~m}, 1 \mathrm{H})$, $7.47 \sim 7.34(\mathrm{~m}, 2 \mathrm{H}), 7.04(\mathrm{dd}, J=8.7,2.4 \mathrm{~Hz}, 1 \mathrm{H}), 6.94(\mathrm{~d}$, $J=2.4 \mathrm{~Hz}, 1 \mathrm{H}), 3.92(\mathrm{~s}, 3 \mathrm{H}), 2.51 \sim 2.35(\mathrm{~m}, 1 \mathrm{H}), 2.05 \sim$ $1.91(\mathrm{~m}, 1 \mathrm{H}), 1.72(\mathrm{~s}, 3 \mathrm{H}), 0.60(\mathrm{t}, J=7.4 \mathrm{~Hz}, 3 \mathrm{H}) ;{ }^{13} \mathrm{C}$ NMR $\left(100 \mathrm{MHz}, \mathrm{CDCl}_{3}\right) \delta: 173.49,162.78,150.27$, $144.34,143.86,131.35,127.89,125.87,125.15,119.48$, 116.35, 115.66, 113.48, 111.86, 55.69, 50.36, 36.69, 28.58, 9.71. HRMS (ESI) calcd for $\mathrm{C}_{19} \mathrm{H}_{19} \mathrm{~N}_{2} \mathrm{O}_{2}[\mathrm{M}+\mathrm{H}]^{+}$ 307.1441, found 307.1449.

5-Ethyl-5-methyl-3-phenylbenzo[4,5]imidazo[2,1-a]isoquinolin-6(5H)-one (2i): White solid. Isolated by flash column chromatography (petroleum ether/ethyl acetate, $V: V=30: 1)$ as eluent to give $2 \mathbf{i}(60 \%$ yield, $72 \mathrm{mg})$. m.p. $151 \sim 152{ }^{\circ} \mathrm{C} ;{ }^{1} \mathrm{H}$ NMR (400 $\left.\mathrm{MHz}, \mathrm{CDCl}_{3}\right) \delta: 8.56$ $(\mathrm{d}, J=8.2 \mathrm{~Hz}, 1 \mathrm{H}), 8.44 \sim 8.36(\mathrm{~m}, 1 \mathrm{H}), 7.86(\mathrm{dd}, J=6.3$, $2.4 \mathrm{~Hz}, 1 \mathrm{H}), 7.73(\mathrm{dd}, J=8.2,1.6 \mathrm{~Hz}, 1 \mathrm{H}), 7.69 \sim 7.63(\mathrm{~m}$, $3 \mathrm{H}), 7.54 \sim 7.41(\mathrm{~m}, 5 \mathrm{H}), 2.55 \sim 2.44(\mathrm{~m}, 1 \mathrm{H}), 2.17 \sim 2.03$ $(\mathrm{m}, 1 \mathrm{H}), 1.80(\mathrm{~s}, 3 \mathrm{H}), 0.64(\mathrm{t}, J=7.4 \mathrm{~Hz}, 3 \mathrm{H}) ;{ }^{13} \mathrm{C} \mathrm{NMR}$ $\left(100 \mathrm{MHz}, \mathrm{CDCl}_{3}\right) \delta: 173.43,149.92,144.85,144.17$, $142.18,140.09,131.38,129.15,128.40,127.34,126.78$, $126.47,126.01,125.65,124.77,122.14,119.81,115.77$, $50.35,36.60,28.50,9.77$. HRMS (ESI) calcd for $\mathrm{C}_{24} \mathrm{H}_{21^{-}}$ $\mathrm{N}_{2} \mathrm{O}[\mathrm{M}+\mathrm{H}]^{+}$353.1648, found 353.1645.

3-Bromo-5-ethyl-5-methylbenzo[4,5]imidazo[2,1- $a$ ]isoquinolin-6(5H)-one (2j): White solid. Isolated by flash column chromatography (petroleum ether/ethyl acetate, $V: V=30: 1)$ as eluent to give $\mathbf{2 j}$ (64\% yield, $68 \mathrm{mg}$ ). m.p. $184 \sim 185{ }^{\circ} \mathrm{C} ;{ }^{1} \mathrm{H}$ NMR (400 MHz, $\left.\mathrm{CDCl}_{3}\right) \delta: 8.39 \sim$ $8.21(\mathrm{~m}, 2 \mathrm{H}), 7.89 \sim 7.76(\mathrm{~m}, 1 \mathrm{H}), 7.65 \sim 7.59(\mathrm{~m}, 2 \mathrm{H})$, $7.49 \sim 7.37(\mathrm{~m}, 2 \mathrm{H}), 2.51 \sim 2.38(\mathrm{~m}, 1 \mathrm{H}), 2.05 \sim 1.92(\mathrm{~m}$, $1 \mathrm{H}), 1.73(\mathrm{~s}, 3 \mathrm{H}), 0.60(\mathrm{t}, J=7.4 \mathrm{~Hz}, 3 \mathrm{H}) ;{ }^{13} \mathrm{C} \mathrm{NMR}(100$ $\left.\mathrm{MHz}, \mathrm{CDCl}_{3}\right) \delta: 172.62,149.19,144.07,143.54,131.30$, $129.43,127.39,126.62,126.17,125.94,122.40,119.97$, $115.81,50.23,36.55,28.32,9.71$. HRMS (ESI) calcd for $\mathrm{C}_{18} \mathrm{H}_{16} \mathrm{BrN}_{2} \mathrm{O}[\mathrm{M}+\mathrm{H}]^{+}$355.0441, found 355.0449.

5-Ethyl-3-iodo-5-methylbenzo[4,5]imidazo[2,1-a]isoquinolin-6(5H)-one (2k): White solid. Isolated by flash column chromatography (petroleum ether/ethyl acetate, $V: V=40: 1)$ as eluent to give $2 \mathbf{k}(52 \%$ yield, $62 \mathrm{mg})$. m.p. $226 \sim 227{ }^{\circ} \mathrm{C} ;{ }^{1} \mathrm{H}$ NMR $\left(400 \mathrm{MHz}, \mathrm{CDCl}_{3}\right) \delta: 8.41 \sim$ $8.32(\mathrm{~m}, 1 \mathrm{H}), 8.18(\mathrm{~d}, J=8.2 \mathrm{~Hz}, 1 \mathrm{H}), 7.87 \sim 7.73(\mathrm{~m}$, $3 \mathrm{H}), 7.50 \sim 7.39(\mathrm{~m}, 2 \mathrm{H}), 2.50 \sim 2.38(\mathrm{~m}, 1 \mathrm{H}), 2.05 \sim 1.92$ $(\mathrm{m}, 1 \mathrm{H}), 1.72(\mathrm{~s}, 3 \mathrm{H}), 0.60(\mathrm{t}, J=7.4 \mathrm{~Hz}, 3 \mathrm{H}) ;{ }^{13} \mathrm{C} \mathrm{NMR}$ $\left(100 \mathrm{MHz}, \mathrm{CDCl}_{3}\right) \delta: 172.54,149.32,144.06,143.41$, $137.10,135.41,131.36,127.21,126.17,125.96,122.92$, $119.99,115.81,98.81,50.04,36.54,28.30,9.72$. HRMS (ESI) calcd for $\mathrm{C}_{18} \mathrm{H}_{16} \mathrm{IN}_{2} \mathrm{O}[\mathrm{M}+\mathrm{H}]^{+}$403.0302, found 403.0303.

5-Ethyl-5-methyl-3-(trifluoromethyl)benzo[4,5]imidazo[2,1-a]isoquinolin-6(5H)-one (2l): White solid. Isolated by flash column chromatography (petroleum ether/ethyl acetate, $V: V=40: 1)$ as eluent to give 21 (76\% yield, 78 mg). m.p. $163 \sim 164{ }^{\circ} \mathrm{C} ;{ }^{1} \mathrm{H}$ NMR (400 MHz, $\left.\mathrm{CDCl}_{3}\right) \delta$ : $8.61(\mathrm{~d}, J=8.2 \mathrm{~Hz}, 1 \mathrm{H}), 8.43 \sim 8.35(\mathrm{~m}, 1 \mathrm{H}), 7.89 \sim 7.82$ $(\mathrm{m}, 1 \mathrm{H}), 7.75(\mathrm{~d}, J=8.4 \mathrm{~Hz}, 1 \mathrm{H}), 7.71(\mathrm{~s}, 1 \mathrm{H}), 7.52 \sim 7.44$ $(\mathrm{m}, 2 \mathrm{H}), 2.55 \sim 2.43(\mathrm{~m}, 1 \mathrm{H}), 2.11 \sim 1.98(\mathrm{~m}, 1 \mathrm{H}), 1.77(\mathrm{~s}$, $3 \mathrm{H}), 0.60$ (t, J=7.4 Hz, 3H); $\left.{ }^{13} \mathrm{C} \mathrm{NMR} \mathrm{(100} \mathrm{MHz,} \mathrm{CDCl}_{3}\right)$ $\delta: 172.52,148.55,144.11,142.35,133.55$ (d, $J=32.8 \mathrm{~Hz})$, $131.43,126.61,126.39,126.34,124.71(\mathrm{~d}, J=3.6 \mathrm{~Hz})$, $123.80(\mathrm{~d}, J=272.9 \mathrm{~Hz}), 123.28$ (d, $J=4.0 \mathrm{~Hz}), 120.31$, 115.94, 50.43, 36.58, 28.23, 9.68. HRMS (ESI) calcd for $\mathrm{C}_{19} \mathrm{H}_{16} \mathrm{~F}_{3} \mathrm{~N}_{2} \mathrm{O}[\mathrm{M}+\mathrm{H}]^{+}$345.1209, found 345.1201.

Methyl 5-ethyl-5-methyl-6-oxo-5,6-dihydrobenzo[4,5]imidazo [2,1-a] isoquinoline-3-carboxylate (2m): White solid. Isolated by flash column chromatography (petroleum ether/ethyl acetate $=20: 1)$ as eluent to give $\mathbf{2 m}(83 \%$ yield, $83 \mathrm{mg}$ ). m.p. $168 \sim 169{ }^{\circ} \mathrm{C}$; ${ }^{1} \mathrm{H}$ NMR (400 MHz, $\left.\mathrm{CDCl}_{3}\right) \delta: 8.54(\mathrm{~d}, J=8.2 \mathrm{~Hz}, 1 \mathrm{H}), 8.42 \sim 8.31(\mathrm{~m}, 1 \mathrm{H})$, $8.19 \sim 8.07(\mathrm{~m}, 2 \mathrm{H}), 7.89 \sim 7.77(\mathrm{~m}, 1 \mathrm{H}), 7.51 \sim 7.37(\mathrm{~m}$, $2 \mathrm{H}), 3.97(\mathrm{~s}, 3 \mathrm{H}), 2.55 \sim 2.39(\mathrm{~m}, 1 \mathrm{H}), 2.15 \sim 2.02(\mathrm{~m}$, 1H), 1.77 (s, 3H), $0.57(\mathrm{t}, J=7.4 \mathrm{~Hz}, 3 \mathrm{H}) ;{ }^{13} \mathrm{C}$ NMR $(100$ $\left.\mathrm{MHz}, \mathrm{CDCl}_{3}\right) \delta 172.92,166.31,148.94,144.09,141.77$, $132.97,131.37,128.64,127.70,127.11,126.25,126.22$, 126.01, 120.19, 115.88, 52.69, 50.33, 36.47, 28.29, 9.69. HRMS (ESI) calcd for $\mathrm{C}_{20} \mathrm{H}_{19} \mathrm{~N}_{2} \mathrm{O}_{3}[\mathrm{M}+\mathrm{H}]^{+} 335.1390$, found 335.1393.

5-Ethyl-5-methyl-3-(methylsulfonyl)benzo[4,5]imidazo[2,1- $a]$ isoquinolin-6(5H)-one (2n): White solid. Isolated by flash column chromatography (petroleum ether/ethyl acetate, $V: V=5: 1)$ as eluent to give 2 n $(81 \%$ yield, 86 mg). m.p. $170 \sim 171{ }^{\circ} \mathrm{C}$; ${ }^{1} \mathrm{H}$ NMR (400 MHz, $\left.\mathrm{CDCl}_{3}\right) \delta$ : $8.69(\mathrm{~d}, J=8.2 \mathrm{~Hz}, 1 \mathrm{H}), 8.39(\mathrm{dd}, J=6.1,3.0 \mathrm{~Hz}, 1 \mathrm{H})$, $8.11 \sim 7.99(\mathrm{~m}, 2 \mathrm{H}), 7.86(\mathrm{dd}, J=6.0,3.0 \mathrm{~Hz}, 1 \mathrm{H}), 7.55 \sim$ $7.42(\mathrm{~m}, 2 \mathrm{H}), 3.15(\mathrm{~s}, 3 \mathrm{H}), 2.55 \sim 2.43(\mathrm{~m}, 1 \mathrm{H}), 2.13 \sim$ $2.02(\mathrm{~m}, 1 \mathrm{H}), 1.79(\mathrm{~s}, 3 \mathrm{H}), 0.59(\mathrm{t}, J=7.4 \mathrm{~Hz}, 3 \mathrm{H}) ;{ }^{13} \mathrm{C}$ NMR $\left(100 \mathrm{MHz}, \mathrm{CDCl}_{3}\right) \delta: 172.17,147.97,144.05$, $143.21,142.97,131.39,128.19,127.17,126.74,126.51$, $125.55,120.46,115.97,50.60,44.59,36.52,28.17,9.73$. HRMS (ESI) calcd for $\mathrm{C}_{19} \mathrm{H}_{19} \mathrm{~N}_{2} \mathrm{O}_{3} \mathrm{~S}[\mathrm{M}+\mathrm{H}]^{+} 355.1111$, found 355.1118 .

4-Ethyl-4-methylbenzo[4,5]imidazo[1,2-a] thieno[2,3-c]pyridin-5(4H)-one (2o): Colorless oil. Isolated by flash column chromatography (petroleum ether/ethyl acetate, $V: V=30: 1)$ as eluent to give $20(63 \%$ yield, $53 \mathrm{mg}) .{ }^{1} \mathrm{H}$ NMR $\left(400 \mathrm{MHz}, \mathrm{CDCl}_{3}\right) \delta: 8.36 \sim 8.30(\mathrm{~m}, 1 \mathrm{H}), 7.79 \sim$ $7.73(\mathrm{~m}, 1 \mathrm{H}), 7.61(\mathrm{~d}, J=5.1 \mathrm{~Hz}, 1 \mathrm{H}), 7.44 \sim 7.36(\mathrm{~m}$, $2 \mathrm{H}), 7.07(\mathrm{~d}, J=5.1 \mathrm{~Hz}, 1 \mathrm{H}), 2.46 \sim 2.33(\mathrm{~m}, 1 \mathrm{H}), 2.01 \sim$ $1.89(\mathrm{~m}, 1 \mathrm{H}), 1.68(\mathrm{~s}, 3 \mathrm{H}), 0.62(\mathrm{t}, J=7.4 \mathrm{~Hz}, 3 \mathrm{H}) ;{ }^{13} \mathrm{C}$ NMR $\left(100 \mathrm{MHz}, \mathrm{CDCl}_{3}\right) \delta: 174.07,147.90,146.99$, $144.03,131.04,131.01,130.82,125.93,125.60,124.08$, 119.73, 115.34, 50.34, 35.96, 27.80, 9.78. HRMS (ESI) calcd for $\mathrm{C}_{16} \mathrm{H}_{15} \mathrm{~N}_{2} \mathrm{OS}[\mathrm{M}+\mathrm{H}]^{+}$283.0900, found 283.0908.

7-Ethyl-7-methylbenzo[ $h]$ benzo[4,5]imidazo[2,1- $a$ ] isoquinolin-8(7H)-one (2p): Yellow solid. Isolated by flash column chromatography (petroleum ether/ethyl acetate, $V: V=30: 1)$ as eluent to give 2 p ( $82 \%$ yield, $80 \mathrm{mg}$ ). m.p. $173 \sim 174{ }^{\circ} \mathrm{C} ;{ }^{1} \mathrm{H}$ NMR $\left(400 \mathrm{MHz}, \mathrm{CDCl}_{3}\right) \delta: 10.53$ $(\mathrm{d}, J=8.8 \mathrm{~Hz}, 1 \mathrm{H}), 8.54 \sim 8.40(\mathrm{~m}, 1 \mathrm{H}), 8.04(\mathrm{~d}, J=8.7$ $\mathrm{Hz}, 1 \mathrm{H}), 7.99 \sim 7.88(\mathrm{~m}, 2 \mathrm{H}), 7.86 \sim 7.78(\mathrm{~m}, 1 \mathrm{H}), 7.64(\mathrm{t}$, 
$J=7.4 \mathrm{~Hz}, 1 \mathrm{H}), 7.57(\mathrm{~d}, J=8.7 \mathrm{~Hz}, 1 \mathrm{H}), 7.53 \sim 7.44(\mathrm{~m}$, $2 \mathrm{H}), 2.60 \sim 2.48(\mathrm{~m}, 1 \mathrm{H}), 2.21 \sim 2.09(\mathrm{~m}, 1 \mathrm{H}), 1.81(\mathrm{~s}$, $3 \mathrm{H}), 0.57$ (t, $J=7.4 \mathrm{~Hz}, 3 \mathrm{H}) ;{ }^{13} \mathrm{C}$ NMR $\left(100 \mathrm{MHz}, \mathrm{CDCl}_{3}\right)$ $\delta: 173.56,150.23,144.22,142.28,132.87,132.80,130.34$, $128.79,128.48,128.13,126.88,125.94,125.89,123.16$, $120.21,118.58,115.84,50.40,36.19,28.65,9.72$. HRMS (ESI) calcd for $\mathrm{C}_{22} \mathrm{H}_{19} \mathrm{~N}_{2} \mathrm{O}[\mathrm{M}+\mathrm{H}]^{+}$327.1492, found 327.1498 .

5-Ethyl-1,3,5-trimethylbenzo[4,5]imidazo[2,1- $a$ ]isoquinolin-6(5H)-one $(\mathbf{2 q})$ : White solid. Isolated by flash column chromatography (petroleum ether/ethyl acetate, $V$ : $V=30: 1)$ as eluent to give $\mathbf{2 q}(67 \%$ yield, $61 \mathrm{mg})$. m.p. $104 \sim 105{ }^{\circ} \mathrm{C} ;{ }^{1} \mathrm{H}$ NMR $\left(400 \mathrm{MHz}, \mathrm{CDCl}_{3}\right) \delta: 8.43 \sim 8.35$ $(\mathrm{m}, 1 \mathrm{H}), 7.86 \sim 7.77(\mathrm{~m}, 1 \mathrm{H}), 7.46 \sim 7.36(\mathrm{~m}, 2 \mathrm{H}), 7.13(\mathrm{~s}$, $2 \mathrm{H}), 3.01(\mathrm{~s}, 3 \mathrm{H}), 2.45 \sim 2.35(\mathrm{~m}, 4 \mathrm{H}), 2.05 \sim 1.94(\mathrm{~m}$, $1 \mathrm{H}), 1.71(\mathrm{~s}, 3 \mathrm{H}), 0.58(\mathrm{t}, J=7.4 \mathrm{~Hz}, 3 \mathrm{H}) ;{ }^{13} \mathrm{C}$ NMR $(100$ $\left.\mathrm{MHz}, \mathrm{CDCl}_{3}\right) \delta: 173.80,150.51,144.37,142.87,140.92$, $139.58,132.09,130.55,125.59,125.42,124.60,119.98$, $119.40,115.73,49.95,36.96,28.80,24.62,21.79,9.73$. HRMS (ESI) calcd for $\mathrm{C}_{20} \mathrm{H}_{21} \mathrm{~N}_{2} \mathrm{O}[\mathrm{M}+\mathrm{H}]^{+} 305.1648$, found 305.1640 .

1,3-Dichloro-5-ethyl-5-methylbenzo[4,5]imidazo[2,1-a]isoquinolin-6(5H)-one (2r): White solid. Isolated by flash column chromatography (petroleum ether/ethyl acetate, $V: V=30: 1)$ as eluent to give $2 \mathbf{r}(55 \%$ yield, $57 \mathrm{mg})$. m.p. $167 \sim 168{ }^{\circ} \mathrm{C} ;{ }^{1} \mathrm{H}$ NMR $\left(400 \mathrm{MHz}, \mathrm{CDCl}_{3}\right) \delta: 8.38$ (dd, $J=6.2,2.8 \mathrm{~Hz}, 1 \mathrm{H}), 7.91(\mathrm{dd}, J=6.3,2.7 \mathrm{~Hz}, 1 \mathrm{H})$, $7.58(\mathrm{~d}, J=1.8 \mathrm{~Hz}, 1 \mathrm{H}), 7.53 \sim 7.42(\mathrm{~m}, 2 \mathrm{H}), 7.40(\mathrm{~d}, J=$ $1.7 \mathrm{~Hz}, 1 \mathrm{H}), 2.50 \sim 2.35(\mathrm{~m}, 1 \mathrm{H}), 2.02 \sim 1.90(\mathrm{~m}, 1 \mathrm{H}), 1.74$ $(\mathrm{s}, 3 \mathrm{H}), 0.61(\mathrm{t}, J=7.4 \mathrm{~Hz}, 3 \mathrm{H}) ;{ }^{13} \mathrm{C} \mathrm{NMR}(100 \mathrm{MHz}$, $\left.\mathrm{CDCl}_{3}\right) \delta: 171.79,146.61,145.80,143.94,136.95,134.42$, $131.23,130.42,126.63,126.15,125.43,120.86,120.20$, $115.72,50.50,37.02,28.32,9.64$. HRMS (ESI) calcd for $\mathrm{C}_{18} \mathrm{H}_{15} \mathrm{Cl}_{2} \mathrm{~N}_{2} \mathrm{O}[\mathrm{M}+\mathrm{H}]^{+}$345.0556, found 345.0559.

\subsection{General procedure for the synthesis of 3}

2-Methyl-1-(2-phenyl-1H-benzo[ $d]$ imidazol-1-yl)prop2-en-1-one (1a): (78.7 mg, $0.3 \mathrm{mmol})$ and BPO (145.3 mg, 2.0 equiv.) were mixtured in $3 \mathrm{~mL} \mathrm{CH}_{3} \mathrm{NO}_{2}$, and then the mixture was refluxed at $120{ }^{\circ} \mathrm{C}$ in oil bath for $2 \mathrm{~h}$. After the completion of the reaction, the mixture was quenched by $\mathrm{NaHCO}_{3}$ (sat. aq. $15 \mathrm{~mL}$ ) and extracted with $\mathrm{CH}_{2} \mathrm{Cl}_{2}(5$ $\mathrm{mL} \times 3)$. Then the organic solvent was concentrated in vacuo. The residue was purified by flash column chromatography with ethyl acetate and petroleum ether as eluent to give 3a.

5-Benzyl-5-methylbenzo[4,5]imidazo[2,1-a]isoquinolin6(5H)-one (3a): White solid. Isolated by flash column chromatography (petroleum ether/ethyl acetate, $V: V=$ $30: 1)$ as eluent to give $\mathbf{3 a}(60 \%$ yield, $61 \mathrm{mg})$. m.p. $147 \sim 148{ }^{\circ} \mathrm{C} ;{ }^{1} \mathrm{H}$ NMR (400 MHz, $\left.\mathrm{CDCl}_{3}\right) \delta: 8.37 \sim 8.31$ $(\mathrm{m}, 1 \mathrm{H}), 8.28(\mathrm{~d}, J=7.7 \mathrm{~Hz}, 1 \mathrm{H}), 7.70 \sim 7.65(\mathrm{~m}, 1 \mathrm{H})$, $7.64 \sim 7.55(\mathrm{~m}, 2 \mathrm{H}), 7.50 \sim 7.43(\mathrm{~m}, 1 \mathrm{H}), 7.42 \sim 7.33(\mathrm{~m}$, $2 \mathrm{H}), 6.89(\mathrm{t}, J=7.4 \mathrm{~Hz}, 1 \mathrm{H}), 6.81(\mathrm{t}, J=7.4 \mathrm{~Hz}, 2 \mathrm{H}), 6.53$ $(\mathrm{d}, J=7.3 \mathrm{~Hz}, 2 \mathrm{H}), 3.56(\mathrm{~d}, J=13.0 \mathrm{~Hz}, 1 \mathrm{H}), 3.17(\mathrm{~d}, J=$ $13.0 \mathrm{~Hz}, 1 \mathrm{H}), 1.94(\mathrm{~s}, 3 \mathrm{H}) ;{ }^{13} \mathrm{C} \mathrm{NMR}\left(100 \mathrm{MHz}, \mathrm{CDCl}_{3}\right) \delta$ : $172.74,149.60,143.86,140.76,135.09,131.60,131.13$,
$129.07,127.95,127.87,127.15,126.70,125.79,125.73$, $125.52,123.65,119.72,115.51,51.20,50.95,26.09$. HRMS (ESI) calcd for $\mathrm{C}_{23} \mathrm{H}_{19} \mathrm{~N}_{2} \mathrm{O}[\mathrm{M}+\mathrm{H}]^{+} 339.1492$, found 339.1493 .

5-Benzyl-1,5-dimethylbenzo[4,5]imidazo[2,1-a]isoquinolin-6(5H)-one (3b): White solid. Isolated by flash column chromatography (petroleum ether/ethyl acetate, $V$ : $V=40: 1)$ as eluent to give $\mathbf{3 b}(61 \%$ yield, $64 \mathrm{mg})$. m.p. $112 \sim 113{ }^{\circ} \mathrm{C} ;{ }^{1} \mathrm{H}$ NMR $\left(400 \mathrm{MHz}, \mathrm{CDCl}_{3}\right) \delta: 8.37(\mathrm{~d}, J=$ $7.6 \mathrm{~Hz}, 1 \mathrm{H}), 7.71(\mathrm{~d}, J=7.4 \mathrm{~Hz}, 1 \mathrm{H}), 7.45(\mathrm{~d}, J=3.9 \mathrm{~Hz}$, 2H), 7.39 (dd, $J=6.6,5.5 \mathrm{~Hz}, 2 \mathrm{H}), 7.30(\mathrm{t}, J=4.2 \mathrm{~Hz}$, $1 \mathrm{H}), 6.90(\mathrm{t}, J=7.0 \mathrm{~Hz}, 1 \mathrm{H}), 6.79(\mathrm{t}, J=7.5 \mathrm{~Hz}, 2 \mathrm{H}), 6.50$ $(\mathrm{d}, J=7.8 \mathrm{~Hz}, 2 \mathrm{H}), 3.51(\mathrm{~d}, J=13.0 \mathrm{~Hz}, 1 \mathrm{H}), 3.13(\mathrm{~d}, J=$ $13.0 \mathrm{~Hz}, 1 \mathrm{H}), 2.90(\mathrm{~s}, 3 \mathrm{H}), 1.94(\mathrm{~s}, 3 \mathrm{H}) ;{ }^{13} \mathrm{C}$ NMR $(100$ $\left.\mathrm{MHz}, \mathrm{CDCl}_{3}\right) \delta: 172.84,149.77,144.04,141.76,139.49$, $135.24,131.17,130.34,130.27,129.16,127.71,127.09$, $125.55,125.46,124.47,122.45,120.01,115.50,51.51$, 51.11, 26.15, 24.45. HRMS (ESI) calcd for $\mathrm{C}_{24} \mathrm{H}_{21} \mathrm{~N}_{2} \mathrm{O}$ $[\mathrm{M}+\mathrm{H}]^{+}$353.1648, found 353.1643.

5-Benzyl-1-methoxy-5-methylbenzo[4,5]imidazo[2,1-a]isoquinolin-6(5H)-one (3c): White solid. Isolated by flash column chromatography (petroleum ether/ethyl acetate, $V: V=10: 1)$ as eluent to give $3 \mathrm{c}(76 \%$ yield, $84 \mathrm{mg})$. m.p. $163 \sim 164{ }^{\circ} \mathrm{C} ;{ }^{1} \mathrm{H}$ NMR $\left(400 \mathrm{MHz}, \mathrm{CDCl}_{3}\right) \delta: 8.34$ $(\mathrm{d}, J=7.0 \mathrm{~Hz}, 1 \mathrm{H}), 7.75(\mathrm{~d}, J=6.9 \mathrm{~Hz}, 1 \mathrm{H}), 7.54(\mathrm{t}, J=8.1$ $\mathrm{Hz}, 1 \mathrm{H}), 7.44 \sim 7.30(\mathrm{~m}, 2 \mathrm{H}), 7.22(\mathrm{~d}, J=7.9 \mathrm{~Hz}, 1 \mathrm{H})$, $7.02(\mathrm{~d}, J=8.3 \mathrm{~Hz}, 1 \mathrm{H}), 6.88(\mathrm{t}, J=7.3 \mathrm{~Hz}, 1 \mathrm{H}), 6.78(\mathrm{t}$, $J=7.5 \mathrm{~Hz}, 2 \mathrm{H}), 6.52(\mathrm{~d}, J=7.3 \mathrm{~Hz}, 2 \mathrm{H}), 4.05$ (s, 3H), 3.53 (d, $J=13.0 \mathrm{~Hz}, 1 \mathrm{H}), 3.14$ (d, $J=13.0 \mathrm{~Hz}, 1 \mathrm{H}), 1.93$ (s, $3 \mathrm{H}) ;{ }^{13} \mathrm{C} \mathrm{NMR}\left(100 \mathrm{MHz}, \mathrm{CDCl}_{3}\right) \delta: 172.63,158.47$, $147.67,144.23,143.38,135.03,131.97,130.06,128.96$, $127.85,127.28,125.58,125.42,120.44,119.00,115.25$, $113.12,110.55,56.69,51.38,51.00,26.41$. HRMS (ESI) calcd for $\mathrm{C}_{24} \mathrm{H}_{21} \mathrm{~N}_{2} \mathrm{O}_{2}[\mathrm{M}+\mathrm{H}]^{+}$369.1598, found 369.1599 .

5-Benzyl-3,5-dimethylbenzo[4,5]imidazo[2,1- $a$ ]isoquinolin-6(5H)-one (3d): Yellow solid. Isolated by flash column chromatography (petroleum ether/ethyl acetate, $V$ : $V=20: 1)$ as eluent to give $\mathbf{3 d}(69 \%$ yield, $73 \mathrm{mg})$. m.p. 92 93 ${ }^{\circ} \mathrm{C} ;{ }^{1} \mathrm{H}$ NMR (400 MHz, $\left.\mathrm{CDCl}_{3}\right) \delta: 8.37 \sim 8.28$ $(\mathrm{m}, 1 \mathrm{H}), 8.17(\mathrm{~d}, J=8.0 \mathrm{~Hz}, 1 \mathrm{H}), 7.68(\mathrm{dd}, J=6.0,3.0 \mathrm{~Hz}$, $1 \mathrm{H}), 7.42 \sim 7.34(\mathrm{~m}, 3 \mathrm{H}), 7.32 \sim 7.27(\mathrm{~m}, 1 \mathrm{H}), 6.91(\mathrm{t}, J=$ $7.3 \mathrm{~Hz}, 1 \mathrm{H}), 6.83(\mathrm{t}, J=7.5 \mathrm{~Hz}, 2 \mathrm{H}), 6.55(\mathrm{~d}, J=7.4 \mathrm{~Hz}$, $2 \mathrm{H}), 3.54(\mathrm{~d}, J=13.0 \mathrm{~Hz}, 1 \mathrm{H}), 3.18(\mathrm{~d}, J=13.0 \mathrm{~Hz}, 1 \mathrm{H})$, $2.52(\mathrm{~s}, 3 \mathrm{H}), 1.93(\mathrm{~s}, 3 \mathrm{H}) ;{ }^{13} \mathrm{C} \mathrm{NMR}\left(100 \mathrm{MHz}, \mathrm{CDCl}_{3}\right) \delta$ : $172.88,149.87,143.90,142.12,140.80,135.18,131.10$, $129.71,129.15,129.02,127.85,127.16,127.13,125.72$, 125.29, 121.00, 119.54, 115.44, 51.12, 50.93, 26.02, 22.15. HRMS (ESI) calcd for $\mathrm{C}_{24} \mathrm{H}_{21} \mathrm{~N}_{2} \mathrm{O}[\mathrm{M}+\mathrm{H}]^{+}$353.1648, found 353.1648 .

5-Benzyl-3-methoxy-5-methylbenzo[4,5]imidazo[2,1-a] isoquinolin-6(5H)-one (3e): White solid. Isolated by flash column chromatography (petroleum ether/ethyl acetate, $V: V=25: 1)$ as eluent to give $3 \mathbf{e}(79 \%$ yield, $87 \mathrm{mg})$. m.p. $112 \sim 113{ }^{\circ} \mathrm{C} ;{ }^{1} \mathrm{H}$ NMR $\left(400 \mathrm{MHz}, \mathrm{CDCl}_{3}\right) \delta: 8.30$ $(\mathrm{dd}, J=5.9,3.3 \mathrm{~Hz}, 1 \mathrm{H}), 8.21(\mathrm{~d}, J=8.3 \mathrm{~Hz}, 1 \mathrm{H}), 7.64$ $(\mathrm{dd}, J=5.8,3.2 \mathrm{~Hz}, 1 \mathrm{H}), 7.39 \sim 7.31(\mathrm{~m}, 2 \mathrm{H}), 7.04 \sim 6.98$ 
$(\mathrm{m}, 2 \mathrm{H}), 6.92(\mathrm{t}, J=7.2 \mathrm{~Hz}, 1 \mathrm{H}), 6.84(\mathrm{t}, J=7.5 \mathrm{~Hz}, 2 \mathrm{H})$, $6.59(\mathrm{~d}, J=7.5 \mathrm{~Hz}, 2 \mathrm{H}), 3.92(\mathrm{~s}, 3 \mathrm{H}), 3.53(\mathrm{~d}, J=13.0 \mathrm{~Hz}$, $1 \mathrm{H}), 3.15(\mathrm{~d}, \quad J=13.0 \mathrm{~Hz}, 1 \mathrm{H}), 1.91(\mathrm{~s}, 3 \mathrm{H}) ;{ }^{13} \mathrm{C} \mathrm{NMR}$ $\left(100 \mathrm{MHz}, \mathrm{CDCl}_{3}\right) \delta: 172.77,162.39,149.80,144.03$, $142.84,135.14,131.09,129.18,127.91,127.68,127.18$, $125.68,125.04,119.33,116.60,115.37,113.68,112.34$, $55.70,51.31,50.89$, 26.13. HRMS (ESI) calcd for $\mathrm{C}_{24} \mathrm{H}_{21} \mathrm{~N}_{2} \mathrm{O}_{2}[\mathrm{M}+\mathrm{H}]^{+}$369.1598, found 369.1589.

5-Benzyl-1-fluoro-5-methylbenzo[4,5]imidazo[2,1- $a$ ]isoquinolin-6(5H)-one (3f): White solid. Isolated by flash column chromatography (petroleum ether/ethyl acetate, $V: V=10: 1)$ as eluent to give $\mathbf{3 f}(30 \%$ yield, $32 \mathrm{mg})$. m.p. $144 \sim 145{ }^{\circ} \mathrm{C} ;{ }^{1} \mathrm{H}$ NMR (400 MHz, $\left.\mathrm{CDCl}_{3}\right) \delta: 8.36$ (dd, $J=6.9,1.7 \mathrm{~Hz}, 1 \mathrm{H}), 7.79(\mathrm{dd}, J=6.8,1.7 \mathrm{~Hz}, 1 \mathrm{H})$, $7.61 \sim 7.54(\mathrm{~m}, 1 \mathrm{H}), 7.46 \sim 7.36(\mathrm{~m}, 3 \mathrm{H}), 7.25 \sim 7.18(\mathrm{~m}$, $1 \mathrm{H}), 6.91(\mathrm{t}, J=7.4 \mathrm{~Hz}, 1 \mathrm{H}), 6.81(\mathrm{t}, J=7.5 \mathrm{~Hz}, 2 \mathrm{H}), 6.52$ $(\mathrm{d}, J=7.2 \mathrm{~Hz}, 2 \mathrm{H}), 3.57(\mathrm{~d}, J=13.0 \mathrm{~Hz}, 1 \mathrm{H}), 3.15(\mathrm{~d}, J=$ 13.0 Hz, 1H), 1.95 (s, 3H); $\left.{ }^{13} \mathrm{C} \mathrm{NMR} \mathrm{(100} \mathrm{MHz,} \mathrm{CDCl}_{3}\right) \delta$ : $172.12,160.33(\mathrm{~d}, J=262.3 \mathrm{~Hz}), 144.18,144.16,143.36$, $134.77,132.14(\mathrm{~d}, J=9.6 \mathrm{~Hz}), 130.16,128.99,128.04$, 127.44, 126.01 (d, $J=21.4 \mathrm{~Hz}), 122.65$ (d, $J=3.7 \mathrm{~Hz})$, $120.57,115.80$ (d, $J=21.3 \mathrm{~Hz}), 115.42,113.13$ (d, $J=9.7$ $\mathrm{Hz}$ ), 51.34, 51.20, 26.27. HRMS (ESI) calcd for $\mathrm{C}_{23} \mathrm{H}_{18} \mathrm{~F}-$ $\mathrm{N}_{2} \mathrm{O}[\mathrm{M}+\mathrm{H}]^{+}$357.1398, found 357.1391.

5-Benzyl-1-chloro-5-methylbenzo[4,5]imidazo[2,1- $a$ ]isoquinolin-6(5H)-on (3g): White solid. Isolated by flash column chromatography (petroleum ether/ethyl acetate, $V: V=10: 1)$ as eluent to give $3 \mathrm{~g}$ (46\% yield, $51 \mathrm{mg}$ ). m.p. $142 \sim 143{ }^{\circ} \mathrm{C} ;{ }^{1} \mathrm{H}$ NMR $\left(400 \mathrm{MHz}, \mathrm{CDCl}_{3}\right) \delta: 8.41 \sim$ $8.31(\mathrm{~m}, 1 \mathrm{H}), 7.84 \sim 7.75(\mathrm{~m}, 1 \mathrm{H}), 7.58 \sim 7.51(\mathrm{~m}, 2 \mathrm{H})$, $7.48(\mathrm{~d}, J=7.7 \mathrm{~Hz}, 1 \mathrm{H}), 7.46 \sim 7.37(\mathrm{~m}, 2 \mathrm{H}), 6.92(\mathrm{t}, J=$ $7.4 \mathrm{~Hz}, 1 \mathrm{H}), 6.80$ (t, $J=7.6 \mathrm{~Hz}, 2 \mathrm{H}), 6.49$ (d, $J=7.2 \mathrm{~Hz}$, $2 \mathrm{H}), 3.52(\mathrm{~d}, \quad J=13.0 \mathrm{~Hz}, 1 \mathrm{H}), 3.11(\mathrm{~d}, J=13.0 \mathrm{~Hz}, 1 \mathrm{H})$, $1.95(\mathrm{~s}, 3 \mathrm{H}) ;{ }^{13} \mathrm{C} \mathrm{NMR}\left(100 \mathrm{MHz}, \mathrm{CDCl}_{3}\right) \delta 171.93$, $148.72,143.79,142.58,134.64,131.38,131.06,129.93$, $129.07,128.01,127.36,127.15,126.11,125.98,125.82$, 122.68, 119.84, 115.50, 51.22, 51.02, 25.74. HRMS (ESI) calcd for $\mathrm{C}_{23} \mathrm{H}_{18} \mathrm{ClN}_{2} \mathrm{O}[\mathrm{M}+\mathrm{H}]^{+}$373.1102, found 373.1109 .

5-Benzyl-3-chloro-5-methylbenzo[4,5]imidazo[2,1-a]isoquinolin-6(5H)-one (3h): White solid. Isolated by flash column chromatography (petroleum ether/ethyl acetate, $V: V=10: 1)$ as eluent to give $\mathbf{3 h}(53 \%$ yield, $59 \mathrm{mg})$. m.p. $131 \sim 132{ }^{\circ} \mathrm{C} ;{ }^{1} \mathrm{H}$ NMR (400 $\mathrm{MHz}, \mathrm{CDCl}_{3}$ ) $\delta: 8.32$ $(\mathrm{dd}, J=6.0,2.8 \mathrm{~Hz}, 1 \mathrm{H}), 8.20(\mathrm{~d}, J=8.4 \mathrm{~Hz}, 1 \mathrm{H}), 7.67$ $(\mathrm{dd}, J=6.0,2.8 \mathrm{~Hz}, 1 \mathrm{H}), 7.54(\mathrm{~d}, J=2.0 \mathrm{~Hz}, 1 \mathrm{H}), 7.45 \sim$ $7.34(\mathrm{~m}, 3 \mathrm{H}), 6.91(\mathrm{t}, J=7.4 \mathrm{~Hz}, 1 \mathrm{H}), 6.82(\mathrm{t}, J=7.5 \mathrm{~Hz}$, $2 \mathrm{H}), 6.55(\mathrm{~d}, J=7.3 \mathrm{~Hz}, 2 \mathrm{H}), 3.55(\mathrm{~d}, J=13.1 \mathrm{~Hz}, 1 \mathrm{H})$, $3.13(\mathrm{~d}, J=13.1 \mathrm{~Hz}, 1 \mathrm{H}), 1.92(\mathrm{~s}, 3 \mathrm{H}) ;{ }^{13} \mathrm{C}$ NMR $(100$ $\left.\mathrm{MHz}, \mathrm{CDCl}_{3}\right) \delta: 171.99,148.65,143.81,142.46,137.74$, $134.67,131.07,129.06,128.49,128.00,127.35,127.09$, $126.94,125.95,125.77,122.29,119.82,115.49,51.27$, 51.00, 25.77. HRMS (ESI) calcd for $\mathrm{C}_{23} \mathrm{H}_{18} \mathrm{ClN}_{2} \mathrm{O}[\mathrm{M}+$ $\mathrm{H}]^{+}$373.1102, found 373.1100 .

5-Benzyl-3-bromo-5-methylbenzo[4,5]imidazo[2,1-a]isoquinolin-6(5H)-one (3i): White solid. Isolated by flash column chromatography (petroleum ether/ethyl acetate,
$V: V=10: 1)$ as eluent to give $3 \mathbf{i}(45 \%$ yield, $56 \mathrm{mg})$. m.p. $145 \sim 146{ }^{\circ} \mathrm{C} ;{ }^{1} \mathrm{H}$ NMR (400 MHz, $\left.\mathrm{CDCl}_{3}\right) \delta: 8.38 \sim$ $8.27(\mathrm{~m}, 1 \mathrm{H}), 8.12(\mathrm{~d}, J=8.4 \mathrm{~Hz}, 1 \mathrm{H}), 7.73 \sim 7.63(\mathrm{~m}$, 2H), 7.59 (dd, $J=8.4,1.8 \mathrm{~Hz}, 1 \mathrm{H}), 7.46 \sim 7.33(\mathrm{~m}, 2 \mathrm{H})$, $6.91(\mathrm{t}, J=7.4 \mathrm{~Hz}, 1 \mathrm{H}), 6.83(\mathrm{t}, J=7.5 \mathrm{~Hz}, 2 \mathrm{H}), 6.54(\mathrm{~d}$, $J=7.3 \mathrm{~Hz}, 2 \mathrm{H}), 3.54(\mathrm{~d}, J=13.1 \mathrm{~Hz}, 1 \mathrm{H}), 3.13(\mathrm{~d}, J=13.1$ $\mathrm{Hz}, 1 \mathrm{H}), 1.92(\mathrm{~s}, 3 \mathrm{H}) ;{ }^{13} \mathrm{C} \mathrm{NMR}\left(100 \mathrm{MHz}, \mathrm{CDCl}_{3}\right) \delta$ : $171.93,148.72,143.79,142.58,134.64,131.38,131.06$, $129.93,129.07,128.01,127.36,127.15,126.11,125.98$, 125.82, 122.68, 119.84, 115.50, 51.22, 51.02, 25.74. HRMS (ESI) calcd for $\mathrm{C}_{23} \mathrm{H}_{18} \mathrm{BrN}_{2} \mathrm{O}[\mathrm{M}+\mathrm{H}]^{+}$417.0597, found 417.0591 .

5-Benzyl-5-methyl-6-oxo-5,6-dihydrobenzo[4,5]imidazo[2,1-a]isoquinoline-3-carbonitrile (3j): White solid. Isolated by flash column chromatography (petroleum ether/ ethyl acetate, $V: V=10: 1)$ as eluent to give $\mathbf{3 j}(40 \%$ yield, $43 \mathrm{mg}$ ). m.p. $131 \sim 132{ }^{\circ} \mathrm{C}$; ${ }^{1} \mathrm{H}$ NMR (400 MHz, $\left.\mathrm{CDCl}_{3}\right) \delta: 8.35(\mathrm{dd}, J=7.6,3.3 \mathrm{~Hz}, 2 \mathrm{H}), 7.84(\mathrm{~s}, 1 \mathrm{H}), 7.72$ $(\mathrm{dd}, J=7.7,2.1 \mathrm{~Hz}, 2 \mathrm{H}), 7.51 \sim 7.38(\mathrm{~m}, 2 \mathrm{H}), 6.92(\mathrm{t}, J=$ $7.4 \mathrm{~Hz}, 1 \mathrm{H}), 6.82(\mathrm{t}, J=7.5 \mathrm{~Hz}, 2 \mathrm{H}), 6.49(\mathrm{~d}, J=7.4 \mathrm{~Hz}$, 2H), 3.59 (d, $J=13.2 \mathrm{~Hz}, 1 \mathrm{H}), 3.12(\mathrm{~d}, J=13.2 \mathrm{~Hz}, 1 \mathrm{H})$, $1.96(\mathrm{~s}, 3 \mathrm{H}) ;{ }^{13} \mathrm{C} \mathrm{NMR}\left(100 \mathrm{MHz}, \mathrm{CDCl}_{3}\right) \delta: 171.46$, $147.54,143.81,143.79,141.60,134.28,131.16,131.14$, $130.87,128.99,128.16,127.62,127.59,126.64,126.38$, $120.33,118.25,115.67,114.73,51.27,51.11,25.52$. HRMS (ESI) calcd for $\mathrm{C}_{24} \mathrm{H}_{18} \mathrm{~N}_{3} \mathrm{O}[\mathrm{M}+\mathrm{H}]^{+}$364.1444, found 364.1450 .

Supporting Information ${ }^{1} \mathrm{H}$ NMR and ${ }^{13} \mathrm{C}$ NMR spectra for compounds 2 and 3. The Supporting Information is available free of charge via the Internet at http://sioc-journal.cn/.

\section{References}

[1] (a) Chatgillaloglu, C.; Ingold, K. U.; Nazran, A. S.; Scaiano, J. C. Organometallics 1983, 2, 1332.

(b) Griller, D.; Ingold, K. U. Acc. Chem. Res. 1976, 9, 13.

(c) Katritzky, A. R.; Zerner, M. C.; Karelson, M. M. J. Am. Chem. Soc. 1986, 108, 7213.

(d) Fischer, H.; Radom, L. Angew. Chem., Int. Ed. 2001, 40, 1340. (e) Goddard, J.-P.; Ollivier, C.; Fensterbank, L. Acc. Chem. Res. 2016, 49, 1924.

(f) Wang, L.-L.; Bao, P.-L.; Liu, W.-W.; Liu, S.-T.; Hu, C.-S.; Yue, H.-L.; Yang, D.-S.; Wei, W. Chin. J. Org. Chem. 2018, 38, 3189 (in Chinese).

(王雷雷, 鲍鹏丽, 刘维伟, 刘思朋, 胡昌松, 岳会兰, 杨道山, 魏 伟, 有机化学, 2018, 38, 3189.)

[2] (a) Wille, U. Chem. Rev. 2013, 113, 813.

(b) Liu, D.; Liu, C.; Lei, A.-W. Chem. Asian J. 2015, 10, 2040. (c) Xu, J.; Song, Q.-L. Chin. J. Org. Chem. 2016, 36, 1151 (in Chinese).

(许健, 宋秋玲, 有机化学, 2016, 36, 1151.)

(d) Li, Q.; Li, M.; Shi, S.; Ji, X.; He, C.; Jiang, B.; Hao, W. Chin. J. Org. Chem. 2020, 40, 384 (in Chinese).

(李庆雪, 李梦伟, 时绍青, 季晓霜, 何春兰, 姜波, 郝文娟, 有机 化学, 2020, 40, 384.)

[3] (a) Patil, N. T.; Yamamoto, Y. Chem. Rev. 2008, 108, 3395. (b) Guo, X.-X.; Gu, D.-W.; Wu, Z.-X.; Zhang, W.-B. Chem. Rev. 2015, $115,1622$.

(c) Wu, B.; Yoshikai, N. Org. Biomol. Chem. 2016, 14, 5402.

(d) Pan, F.; Shu, C.; Ye, L.-W. Org. Biomol. Chem. 2016, 14, 9456. 
(e) Alam, K.; Hong, S. W.; Oh, K. H.; Park, J. K. Angew. Chem., Int. Ed. 2017, 56, 13387.

(f) Xie, W.-L.; Wu, Y.-Q.; Zhang, J.-A.; Mei, Q.-H.; Zhang, Y.-H.; Zhu, N.; Liu, R.-Z.; Zhang, H.-L. Eur. J. Med. Chem. 2018, 145, 35. (g) Gong, X.; Li, G.; Gan, Z.; Yan, Q.; Dou, X.; Yang, D. Asian J. Org. Chem. 2019, 8, 1472.

(h) Shi, L.; Xu, J.; B, i J.; Zhang, Z.; Liu, T.; Yang, X.; Zhang, G. Chin. J. Org. Chem. 2018, 38, 3016 (in Chinese)

(李庆雪, 李梦伟, 时绍青, 季晓霜, 何春兰, 姜波, 郝文娟, 有机 化学, 2018, 38, 3016.)

(i) Sun, K.; Si, Y.-F.; Chen, X.-L.; Lv, Q.-Y.; Peng, Y.-Y.; Qu, L.-B. Asian J. Org. Chem. 2019, 8, 2042.

[4] (a) Meng, G.; Niu, H.-Y.; Qu, G.-R.; Fossey, J. S.; Li, J.-P.; Guo, H.-M. Chem. Commun. 2012, 48, 9601.

(b) Iaroshenko, V. O.; Ostrovskyi, D.; Miliutina, M.; Maalik, A.; Villinger, A.; Tolmachev, A.; Volochnyuk, D. M.; Langer, P. Adv. Synth. Catal. 2012, 354, 2495.

(c) Sun, X.; Hu, Y.; Nie, S.-Z.; Yan, Y.-Z.; Zhang, X.-J.; Yan, M. Adv. Synth. Catal. 2013, 355, 2179.

(d) Sun, X.; Lv, X.-H.; Ye, L.-M.; Hu, Y.; Chen, Y.-Y.; Zhang, X.-J.; Yan, M. Org. Biomol. Chem. 2015, 13, 7381.

(e) Liu, Y.; Chen, G.-Q.; Tse, C.-W.; Guan, X.; Xu, Z.-J.; Huang, J.-S.; Che, C.-M. Chem. Asian J. 2015, 10, 100.

(f) Nguyen, T. B.; Ermolenkoa, L.; Al-Mourabi, A. Chem. Commun. 2016, $52,4914$.

[5] (a) Parsons, P. J.; Penkett, C. S.; Shell, A. J. Chem. Rev. 1996, 96, 195.

(b) Wang, Z.; He, W.-M. Chin. J. Org. Chem. 2019, 39, 3594 (in Chinese).

(王峥，何卫民，有机化学, 2019, 39, 3594.)

[6] Mai, S.-Y.; Luo, Y.-X.; Huang, X.-Y.; Shu, Z.-H.; Li, B.-N.; Lan, Y.; Song, Q.-L. Chem. Commun. 2018, 54, 10240.

[7] (a) Sun, K.; Li, S.-J.; Chen, X.-L.; Liu, Y.; Huang, X.-Q.; Wei, D.-H.; Qu, L.-B.; Zhao, Y.-F.; Yu, B. Chem. Commun. 2019, 55, 2861.

(b) Zeng, F.-L.; Sun, K.; Chen, X.-L.; Yuan, X.-Y.; He, S.-Q.; Liu, Y.; Peng, Y.-Y.; Qu, L.-B.; Lv, Q.-Y.; Yu, B. Adv. Synth. Catal. 2019, 361, 5176 .

[8] (a) Barreiro, E. J.; Kümmerle, A. E.; Fraga, C. A. M. Chem. Rev. 2011, 111, 5215 .

(b) Schönherr, H.; Cernak, T. Angew. Chem., Int. Ed. 2013, 52, 12256.

(c) Yan, G.-B.; Borah, A. J.; Wang, L.-G.; Yang, M.-H. Adv. Synth. Catal. 2015, 357, 1333.

(d) Sun, S.-M.; Fu, J.-M. Bioorg. Med. Chem. Lett. 2018, 28, 3283.

(e) Chen, Y.-T. Chem.-Eur. J. 2019, 25, 3405.

[9] Rust, F. F.; Seubold, F. H.; Vaughan, W. E. J. Am. Chem. Soc. 1948, $70,3258$.

[10] (a) Xia, Q.-Q.; Liu, X.-L.; Zhang, Y.-J.; Chen, C.; Chen, W.-Z. Org. Lett. 2013, 15, 3326.

(b) Xu, Z.-B.; Yan, C.-X.; Liu, Z.-Q. A. Org. Lett. 2014, 16, 5670.

(c) Li, G.; Yang, S.-L.; Lv, B.-J.; Han, Q.-Q.; Ma, X.-X.; Sun, K.; Wang, Z.-Y.; Zhao. F.; Lv, Y.-H.; Wu, H.-K. Org. Biomol. Chem. $2015,13,11184$.

(d) Bao, Y.-J.; Yan, Y.-Z.; Xu, K.; Su, J.-H.; Zha, Z.-G.; Wang, Z.-Y. J. Org. Chem. 2015, 80, 4736.

(e) Li, Q.; Li, Y.-R.; Hu, W.-P.; Hu, R.-J.; Li, G.-G.; Lu, H.-J. Chem.Eur. J. 2016, 22, 12286 .

(f) Zhang, P.-Z.; Li, J.-A.; Zhang, L.; Shoberu, A.; Zou, J.-P.; Zhang, W. Green Chem. 2017, 19, 919.

[11] (a) Guo, S.-J.; Wang, Q.; Jiang, Y.; Yu, J.-T. J. Org. Chem. 2014, 79, 11285.

(b) Yang, Y.; Bao, Y.-J.; Guan, Q.-Q.; Sun, Q.; Zha, Z.-G.; Wang, Z.-Y. Green Chem. 2017, 19, 112.

(c) Zhu, N.-B.; Zhao, J.-G.; Bao, H.-L. Chem. Sci. 2017, 8, 2081.
[12] (a) Dai, Q.; Yu, J.-T.; Jiang, Y.; Guo, S.-J.; Yang, H.-T.; Cheng, J. Chem. Commun. 2014, 50, 3865.

(b) Dai, Q.; Yu, J.-T.; Feng, X.-M.; Jiang, Y.; Yang, H.-T.; Cheng, J. Adv. Synth. Catal. 2014, 356, 3341 .

(c) Tan, F.-L.; Song, R.-J.; Hu, M.; Li, J.-H. Org. Lett. 2016, 18, 3198.

(d) Zhuang, H.; Zeng, R.; Zou, J.-P. Chin. J. Chem. 2016, 34, 368.

(e) Bao, X.; Yokoe, T.; Ha, T. M.; Wang, Q.; Zhu, J.-P. Nat. Commun. 2018, 9, 3725 .

(f) Liu, D.; Yu, Y.-Q.; Xia, Z.; Song, Z.-N.; Liao, L.-H.; Tan, Z.; Chen, X. Eur. J. Org. Chem. 2019, 2019, 6930.

[13] (a) Hix, S.; Kadiiska, M. B.; Mason, R. P.; Augusto, O. Chem. Res. Toxicol. 2000, 13, 1056.

(b) Zhu, Y.; Yan, H.; Lu, L.-H.; Liu, D.-F.; Rong, G.-W.; Mao, J.-C. J. Org. Chem. 2013, 78, 9898.

(c) Patel, O. P. S.; Nandwana, N. K.; Sah, A. K.; Kumar, A. Org. Biomol. Chem. 2018, 16, 8620.

[14] (a) Sun, K.; Li, Y.; Xiong, T.; Zhang, J.-P.; Zhang, Q. J. Am. Chem. Soc. 2011, 133, 1694.

(b) Sun, K.; Wang, X.; Liu, L.-L.; Sun, J.-J.; Liu, X.; Li, Z.-D.; Zhang, Z.-G.; Zhang, G.-S. ACS Catal. 2015, 5, 7194.

(c) Sun, K.; Li, Y.; Zhang, Q. Sci. China Chem. 2015, 58, 1354

(d) Wang, X.; Mu, S.-Q.; Sun, T.; Sun, K. Chin. J. Org. Chem. 2019, 39, 2802 (in Chinese).

(王薪, 穆石强, 孙婷, 孙凯, 有机化学, 2019, 39, 2802.)

(e) Sun, K.; Li, Y.-L.; Feng, R.-R.; Mu, S.-Q.; Wang, X.; Zhang, B. J. Org. Chem. 2020, 85, 1001.

[15] (a) Sun, K.; Wang, X.; Li, D.-H.; Zhu, Z.-H.; Jiang, Y.-Q.; Xiao, B.-B. Chem. Commun. 2014, 50, 12880.

(b) Sun, K.; Lv, Y.-H.; Wang, J.-J.; Sun, J.-J.; Liu, L.-L.; Jia, M.-Y.; Liu, X.; Li, Z.-D.; Wang, X. Org. Lett. 2015, 17, 4408.

(c) Sun, K.; Shi, Z.-D.; Liu, Z.-H.; Luan, B.-X.; Zhu, J.-L.; Xue, Y.-R. Org. Lett. 2018, 20, 6687.

(d) Sun, K.; Wang, S.-N.; Feng, R.-R.; Zhang, Y.-X.; Wang, X.; Zhang, Z.-G.; Zhang, B. Org. Lett. 2019, 21, 2052.

(e) Wang, X.; Li, C.-H.; Zhang, Y.-X.; Zhang, B.; Sun, K. Org. Biomol. Chem. 2019, 17, 8364.

(f) Sun, K.; Li, G.-F.; Li, Y.-Y.; Yu, J.; Zhao, Q.; Zhang, Z.-G.; Zhang, G.-S. Adv. Synth. Catal. 2020, DOI: 10.1002/adsc. 202000040.

[16] (a) Liu, Y.-Y.; Xiong, J.; Wei, L.; Wan, J.-P. Adv. Synth. Catal. 2020, $362,877$.

(b) Wan, J.-P.; Tu, Z.; Wang, Y.-Y. Chem.-Eur. J. 2019, 25, 6907.

[17] (a) Hu, M.; Fan, J.-H.; Liu, Y.; Ouyang, X.-H.; Song, R.-J.; Li, J.-H. Angew. Chem., Int. Ed. 2015, 54, 9577.

(b) Hu, M.; Song, R.-J.; Li, J.-H. Angew. Chem., Int. Ed. 2015, 54, 608.

(c) Guo, Y.-H.; Xiang, Y.-F.; Wei, L.; Wan, J.-P. Org. Lett. 2018, 20, 3971.

(d) Wan, C.; Song, R.-J.; Li, J.-H. Org. Lett. 2019, 21, 2800.

(e) Wang, L.-L.; Zhang, Y.-L.; Zhang, M.; Bao, P.-L.; Lv, X.-X.; Liu, H.-G.; Zhao, X.-H.; Li, J.-S.; Luo, Z.-D.; Wei, W. Tetrahedron Lett. 2019, 60, 1845.

(f) Li, G.-Q.; Gan, Z.-Y.; Kong, K.-X.; Dou, X.-M.; Yang, D.-S. Adv. Synth. Catal. 2019, 361, 1808.

(g) Xie, L.-Y.; Fang, T.-G.; Tan, J.-X.; Zhang, B.; Cao, Z.; Yang, L.-H.; He, W.-M., Green Chem. 2019, 21, 3858. S.-J.; Jiang, B. Chem. Commun. 2019, 55, 13231.

(i) Guo, T.; Wei, X.-N.; Liu, Y.; Zhang, P.-K.; Zhao, Y.-H. Org. Chem. (h) Zhang, T.-S.; Zhang, H.-P.; Fu, R.; Wang, J.-Y.; Hao, W.-J.; Tu, Front. 2019, 6, 1414.

(j) Yang, S.-B.; Li, Y.; Zhang, Q. Chin. J. Org. Chem. 2019, 39, 2226 (in Chinese).

(杨胜彪，李燕，张前，有机化学, 2019, 39, 2226.) 